\title{
Efficient Non-Recursive Design of Second-Order Spectral-Null Codes
}

\author{
Luca G. Tallini, Danilo Pelusi, Raffaele Mascella, Laura Pezza, \\ Samir Elmougy, and Bella Bose, Fellow, IEEE
}

\begin{abstract}
A new efficient design of second-order spectralnull (2-OSN) codes is presented. The new codes are obtained by applying the technique used to design parallel decoding balanced (i.e., 1-OSN) codes to the random walk method introduced by some of the authors for designing 2-OSN codes. This gives new non-recursive efficient code designs, which are less redundant than the code designs found in the literature. In particular, if $k \in \mathbb{I N}$ is the length of a 1-OSN code then the new 2-OSN coding scheme has length $n=k+r \in \mathbb{I N}$ with an extra redundancy of $r \simeq 2 \log _{2} k+(1 / 2) \log _{2} \log _{2} k-0.174$ check bits, with $k$ and $r$ even and $n$ multiple of 4 . The whole coding process requires $O(k \log k)$ bit operations and $O(k)$ bit memory elements.
\end{abstract}

Index Terms - High order spectral null codes, balanced codes, Knuth's complementation method, parallel decoding scheme, optical and magnetic recording.

\section{INTRODUCTION}

$\mathbf{L}$ ET $\Phi=\{-1,+1\}$ be the bipolar alphabet. The set of $q$ th-order spectral-null words $\mathcal{S N}(n, q) \subseteq \Phi^{n}$ is defined as [5], [6], [14],

$$
\mathcal{S} \mathcal{N}^{\prime}(n, q) \stackrel{\text { def }}{=}\left\{\begin{array}{l|l}
X \in \Phi^{n} & \begin{array}{l}
m_{i}(X)=0 \\
\text { for all integer } i \in[0, q-1]
\end{array}
\end{array}\right\}
$$

where $m_{i}(X) \stackrel{\text { def }}{=} x_{1} 1^{i}+x_{2} 2^{i}+\ldots+x_{n} n^{i}=\sum_{j=1}^{n} x_{j} j^{i}$ is the $i$ th moment (or, the $m_{i}$-weight) of the word $X=x_{1} x_{2} \ldots$ $x_{n} \in \Phi^{n}$, and sums and products are over the real numbers. Any word in $\mathcal{S N}(n, q)$ is called $q$ th-order spectral-null word (briefly, a $q$-OSN word). In the following, if $m_{i}(X)=0$ then

Manuscript received July 4, 2015; revised March 29, 2016; accepted March 30, 2016. Date of publication April 20, 2016; date of current version May 18, 2016. This work was supported in part by the National Science Foundation within the Division of Computing and Communication Foundations under Grant CCF-1117215 and Grant CCF-1423656, in part by Italian MIUR under Grant PRIN 2009-2009EL7424, in part by the National Plan for Science, Technology and Innovation (MAARIFAH), King Abdulaziz City for Science and Technology, Kingdom of Saudi Arabia, Award Number (10-INF1165-02). This paper was partially presented at the the 2013 IEEE International Symposium on Information Theory.

L. G. Tallini, D. Pelusi, and R. Mascella are with the Facoltà di Scienze della Comunicazione, Università degli Studi di Teramo, Teramo 64100, Italy (e-mail: 1tallini@unite.it; dpelusi@unite.it; rmascellag@unite.it).

L. Pezza is with the Dipartimento di Scienze di Base e Applicate per l'Ingegneria, Università degli Studi di Roma "La Sapienza", Rome 00161, Italy (e-mail: pezza@dmmm.uniroma1.it).

S. Elmougy is with the Department of Computer Science, Faculty of Computers and Information, Mansoura University, Mansoura 35516, Egypt, and also with the Department of Computer Science, College of Computer and Information Sciences, King Saud University, Riyadh 11543, Saudi Arabia (e-mail: mougy@mans.edu.eg).

B. Bose is with the School of Electrical Engineering and Computer Science, Oregon State University, Corvallis, OR 97331 USA (e-mail: bose@eecs.orst.edu).

Communicated by M. Schwartz, Associate Editor for Coding Techniques. Digital Object Identifier 10.1109/TIT.2016.2555322 we will say that the word $X$ is $m_{i}$-balanced. A binary code $\mathcal{C}$ is a $q$ th-order spectral-null code with $\tilde{k}$ information bits and length $n$ (briefly, a $q-\operatorname{OSN}(n, \tilde{k})$ code) if, and only if

1) $\mathcal{C}$ is a subset of $\mathcal{S N}(n, q)$, and

2) $\mathcal{C}$ has $2^{\tilde{k}}$ codewords.

When $q=1$, these codes coincide with the so-called balanced or DC-free block codes [1]-[3], [5], [7], [10]-[14], [17]-[19], [21], [23]-[25], [28]. For values of $q$ greater than 1, the $q$-OSN $(n, \tilde{k})$ codes are considered in digital recording to achieve a better rejection of the low frequency components of the transmitted signal and enhancing the error correction capability of codes used in partial-response channels [6], [14]. The $q$-OSN codes can be also considered over the binary alphabet $\mathbf{Z}_{2}=\{0,1\}$ [20]. In fact, by replacing the symbol -1 with 0 and +1 with 1 , the set $\mathcal{S N}(n, q)$ becomes equivalent to the set $\mathcal{S N}^{\prime}(n, q) \subseteq \mathbf{Z}_{2}^{n}$

$$
\mathcal{S N}^{\prime}(n, q) \stackrel{\text { def }}{=}\left\{\begin{array}{l|l}
X \in \mathbf{Z}_{2}^{n} & \begin{array}{c}
m_{i}(X)=\sum_{j=1}^{n} x_{j} j^{i}=\frac{1}{2} \sum_{j=1}^{n} j^{i}, \\
\text { for all integer } i \in[0, q-1]
\end{array}
\end{array}\right\} ;
$$

where the sums and products are done in the real field $\mathbf{I R}$. Since $\mathcal{S} \mathcal{N}(n, q)$ and $\mathcal{S} \mathcal{N}^{\prime}(n, q)$ are equivalent, in the rest of the paper $\mathcal{S} \mathcal{N}(n, q)$ is used for $\mathcal{S} \mathcal{N}^{\prime}(n, q)$. For example,

$$
\mathcal{S N}(n, 2)=\left\{\begin{array}{l|l}
X \in \mathbf{Z}_{2}^{n} & \begin{array}{l}
m_{0}(X)=\sum_{j=1}^{n} x_{j}=\frac{n}{2} \text { and } \\
m_{1}(X)=\sum_{j=1}^{n} x_{j} j=\frac{n(n+1)}{4}
\end{array}
\end{array}\right\}
$$

and $\mathcal{S N}(n, 2) \neq \emptyset$ if, and only if, $n$ is multiple of 4 [20].

The code design problem is to convert the information words into $q$ th-order spectral-null words using the minimum possible redundancy. For $q=2$, this minimum redundancy is [20]

$$
r_{\min }(k)=2 \log _{2} n-1.141 .
$$

Also, the conversion should be done so that the encoding and decoding processes are as computationally simple as possible. Efficient design of $q$ th-order spectral-null codes for $q=2$, has been considered in [8], [9], [14], [20], [22], [26], [27] and, for $q=3$, in [15].

In this paper we are concerned with designing 2-OSN codes whose encoding and decoding functions can be computed combining the Knuth's optimal refined parallel decoding scheme for balanced codes given in [1], [11], [12], and [19] 
with the random walk method for 2-OSN codes given in [9], [20], and [26]. Here we assume that the information words are already $m_{0}$-balanced words of even length $k$ (i.e., the information words belongs to $\mathcal{S N}(k, 1) \neq \varnothing)$ by using a $1-\operatorname{OSN}(k, \tilde{k})$ code design. Given $k, \mu_{0}, \mu_{1} \in \mathbf{I N}$, let

$$
S\left(k, \mu_{0}\right) \stackrel{\text { def }}{=}\left\{X \in \mathbf{Z}_{2}^{k}: m_{0}(X)=\mu_{0}\right\} \subseteq \mathbf{Z}_{2}^{k}
$$

and

$$
\begin{array}{r}
S\left(k, \mu_{0}, \mu_{1}\right) \stackrel{\text { def }}{=}\left\{X \in \mathbf{Z}_{2}^{k}: m_{0}(X)=\mu_{0} \text { and } m_{1}(X)=\mu_{1}\right\} \\
\subseteq S\left(k, \mu_{0}\right) .
\end{array}
$$

Now, given an $m_{0}$-balanced word $X=x_{1} x_{2} \ldots x_{k} \in S(k, k / 2)$ (i.e., such that $m_{0}(X)=k / 2 \in \mathbf{I N}$ ), the idea is to "walk randomly" towards the reverse, $X \stackrel{R}{\stackrel{\text { def }}{=}} x_{k} x_{k-1} \ldots x_{1}$, of $X$, by exchanging adjacent bits, until a word $X^{\left(d_{h_{b a l}}\right)}$ is reached, with $d_{h}, h, h_{b a l} \in \mathbb{I N}$, where this word satisfies a specific property for its second moment. Note that permutations of adjacent bits in the word $X$ do not alter the value of its first moment, while its second moment may change with a variation of $+1,-1$, or 0 .

Moreover, a family of sets of check words, $\mathcal{C S}$, is built so that each set of check words, $\Gamma_{h} \in \mathcal{C S}$, identifies a specific step $d_{h}$ of the random walk, where $h=0,1, \ldots,|\mathcal{C} \mathcal{S}|-1$. As such, each set $\Gamma_{h}$ of the family of check words defines an encoding function $\left\langle\Gamma_{h}\right\rangle$. And, as the main theorem states (Theorem 2), there always exists at least one step $d_{h_{b a l}}$ such that $X^{\left(d_{h_{b a l}}\right)}=\left\langle\Gamma_{h_{b a l}}\right\rangle(X)$ concatenated with one of the check words identifying $\left\langle\Gamma_{h}\right\rangle$, becomes a 2-OSN word. As in [19] for 1-OSN codes, such possibly many indices $h_{b a l}$, for a given code design, are referred to as the 2-OSN balancing indices of $X$. Here we let $h_{\text {bal }}(X) \subseteq \mathbf{I N}$ indicate the set of all possible balancing indices of $X$. Now, a check word $C=C(X) \in \mathbf{Z}_{2}^{r}$, $r \in \mathbb{I N}$, is appended to obtain a $n=k+r$ bit codeword

$$
\mathcal{E}_{2}(X)=X^{\left(d_{h_{b a l}}\right)} C(X) \in \mathbf{Z}_{2}^{n}
$$

as encoding of $X$. In order to implement this strategy, such check $C$ must be chosen so that the properties 1),2) and 3 ) below hold.

1) The codeword $\mathcal{E}_{2}(X)$ is $m_{0}$-balanced; that is,

$$
m_{0}\left(\mathcal{E}_{2}(X)\right)=m_{0}\left(X^{\left(d_{h_{b a l}}\right)}\right)+m_{0}(C)=\frac{n}{2} \in \mathbf{I N} .
$$

Note that, since $n$ is a multiple of 4 (otherwise, we have $\mathcal{S N}(n, 2)=\emptyset)$, if $k$ is even and $X \in S(k, k / 2)$ then $r$ is even and $C \in S(r, r / 2)$.

2) The codeword $\mathcal{E}_{2}(X)$ is $m_{1}$-balanced; that is,

$$
m_{1}\left(\mathcal{E}_{2}(X)\right)=m_{1}\left(X^{\left(d_{h_{b a l}}\right)} C\right)=\frac{n(n+1)}{4} \in \mathbf{I N} .
$$

Thus, 1) and 2) make $\mathcal{E}_{2}(X)$ a 2nd-order spectral-null word.

3) The original information word $X$ can be recovered from $X^{\left(d_{h_{b a l}}\right)}$ and $C$.

In Section II, to fulfill the above properties, we start with the 1 -OSN words of even length, $k \in 2 \mathbf{I N}$ and then we encode these words into 2-OSN words whose length is $n \in 4 \mathbf{I N}$. This last step is obtained by adapting the coding scheme given in [1] and [19] for balanced codes to fit the random walk coding scheme given in [20] for 2-OSN codes. The combination of the two methods gives efficient non-recursive code designs for any value of the parameters $k \in 2 \mathbf{I N}$, $r=n-k \in 2 \mathbf{I N}$ and $n \in 4 \mathbf{I N}$, provided that

$$
\frac{k(k-1)}{2} \leq\left(\begin{array}{c}
r \\
r / 2
\end{array}\right) \Longleftrightarrow k \leq \frac{1+\sqrt{1+8\left(\begin{array}{c}
r \\
r / 2
\end{array}\right)}}{2} .
$$

Note that, if

$$
k \simeq \frac{1}{2}\left(1+\sqrt{1+8\left(\begin{array}{c}
r \\
r / 2
\end{array}\right)}\right)
$$

then

$$
r \simeq 2 \log _{2} k+(1 / 2) \log _{2} \log _{2} k-0.174
$$

because of Stirling's approximation. As shown in Table II, we get 2-OSN codes which are considerably less redundant than the codes found in the literature. To our knowledge, all code designs found in the literature are recursive and use only 2-OSN words as check words. Here, on the other hand, the code designs are non-recursive and make a potential good use of all possible 1-OSN words as check words. Hence, the information rate improvement has two reasons. First, the nonrecursiveness of the code designs makes sure that any unuseful redundancy does not add up at every encoding recursion step. Second, the presented code designs make available many more check words to use in the design. In any case, the code rate improvement is of the order of $2 \log _{2} \log _{2} k+O(\log \log \log k)$ if $k$ is choosen to be the length of an optimal 1-OSN/balanced code. This can be seen by comparing (3) with the formula for the code length (6) in [20]. In Section III, we show that the whole coding process can be implemented with $O(k \log k)$ bits operations and using $O(k)$ memory bits. In Section IV, some concluding remarks are given.

\section{The Proposed Refined Coding Scheme}

The main idea of the code design is to convert a balanced data word into an "almost 2-OSN" word, using an appropriate function from a set of " $m_{1}$-balancing functions", and append a check word. This check word 1) "encodes" which encoding function is used in the encoding process $\mathcal{E}_{2}$, and 2) corrects any further $m_{1}$-imbalance of the almost 2-OSN word. To decode a codeword, the receiver simply applies, to the $m_{0}$-balanced information part, the inverse of the function encoded by the check part.

The coding scheme is captured by the following concept of the set of $m_{1}$-balancing functions.

Definition 1 (Set of $m_{1}$-Balancing Functions): Let $k$, $r \in 2 \mathbf{I N}$ be given so that $n \stackrel{\text { def }}{=} k+r \in 4 \mathbf{I N}$. Also, let

$$
\mathcal{C S} \stackrel{\text { def }}{=}\left\{\Gamma_{0}, \Gamma_{1}, \ldots, \Gamma_{p-1}\right\},
$$

be a family of $p \in \mathbf{I N}$ non-empty subsets of the set of all the $r$-bit $m_{0}$-balanced check words $S(r, r / 2)$. Also, for all indices $h \in[0, p-1]$, let

$$
\left\langle\Gamma_{h}\right\rangle: S(k, k / 2) \rightarrow S(k, k / 2)
$$


indicate a function from the set of all $k$-bit $m_{0}$-balanced data words, $S(k, k / 2)$, into itself. We say that the set of functions

$$
\mathcal{B} \stackrel{\text { def }}{=}\left\{\left\langle\Gamma_{h}\right\rangle: h \in[0, p-1]\right\}
$$

is a set of $m_{1}$-balancing functions if, and only if, the following conditions hold.

1) The sets $\Gamma_{h}$ are pair-wise disjoint; i.e.,

$$
\Gamma_{i} \cap \Gamma_{j}=\emptyset \Longleftrightarrow i \neq j
$$

Thus, from $C \in \Gamma_{h}$ it is possible to unambiguously recover $h \in[0, p-1]$.

2) For every $m_{0}$-balanced information word $X \in S(k, k / 2)$ there exists one $h_{b a l} \in[0, p-1]$ and $C_{h_{b a l}} \in \Gamma_{h_{b a l}}$ such that

$$
m_{1}\left(\left\langle\Gamma_{h_{b a l}}\right\rangle(X) C_{h_{b a l}}\right)=\frac{n(n+1)}{4} .
$$

In the sequel, we refer to an index $h_{b a l}$ as an " $m_{1}$-balancing index" of $X$ and we let $h_{b a l}(X) \subseteq$ $[0, p-1]$ indicate the set of all possible balancing indices of $X$. Note that, for all $X \in S(k, k / 2)$,

$$
\begin{aligned}
m_{0}\left(\left\langle\Gamma_{h_{\text {bal }}}\right\rangle(X) C_{h_{\text {bal }}}\right) & =m_{0}\left(\left\langle\Gamma_{h_{\text {bal }}}\right\rangle(X)\right)+m_{0}\left(C_{h_{\text {bal }}}\right) \\
& =\frac{k}{2}+\frac{r}{2}=\frac{n}{2} .
\end{aligned}
$$

3) For all indices $h \in[0, p-1]$, the function $\left\langle\Gamma_{h}\right\rangle$ is one-to-one so that, from $h$ and $Y=\left\langle\Gamma_{h}\right\rangle(X)$ it is possible to unambiguously recover $X$.

Given a set of $m_{1}$-balancing functions, every $m_{0}$-balanced data word $X \in S(k, k / 2)$ is encoded as

$$
\mathcal{E}_{2}(X)=\left\langle\Gamma_{h_{b a l}}\right\rangle(X) C_{h_{b a l}}
$$

where $h_{b a l} \in h_{b a l}(X)$ is an " $m_{1}$-balancing index" of $X$; for example, the smallest index in the set $h_{b a l}(X)$. We readily note that, to give a well defined set of $m_{1}$-balancing functions $\mathcal{B}$, it is required to define

a) the partition $\mathcal{C S}$, and

b) each injective function $\left\langle\Gamma_{h}\right\rangle: S(k, k / 2) \rightarrow S(k, k / 2)$ in $\mathcal{B}$;

and these two requirements are dependent on one another by the condition 2) of Definition 1. Now, a possible construction for such balancing functions $\langle\Gamma\rangle \in \mathcal{B}$, with $\Gamma \in \mathcal{C} \mathcal{S}$, is explained below.

First, given

$$
X=x_{1} x_{2} x_{3} \ldots x_{i} \ldots x_{j} \ldots x_{k-1} x_{k}
$$

let

$$
X^{(i, j)}=x_{1} x_{2} x_{3} \ldots \underline{x_{j}} \ldots \underline{x_{i}} \ldots x_{k-1} x_{k}
$$

be the word obtained form $X$ by exchanging the $i$ th bit with the $j$ th bit, and

$$
X^{R}=\underline{x_{k} x_{k-1} \ldots x_{j} \ldots x_{i} \ldots x_{3} x_{2} x_{1}}
$$

be the reverse of $X$. The code design is based on the following observations [20].

Observation 1: The $m_{0}$-weight, $m_{0}(X)$, does not change if the bits of $X$ are permuted.
Observation 2: The relation

$$
m_{1}(X)+m_{1}\left(X^{R}\right)=(k+1) m_{0}(X)
$$

holds. So, if $m_{0}(X)=k / 2 \in \mathbf{I N}$ then

$$
m_{1}(X) \leq \frac{k(k+1)}{4} \Longleftrightarrow m_{1}\left(X^{R}\right) \geq \frac{k(k+1)}{4} .
$$

Observation 3: The following relation holds

$$
m_{1}\left(X^{(i, i+1)}\right)=m_{1}(X)+\left(x_{i}-x_{i+1}\right) .
$$

This implies that by exchanging two consecutive bits of $X$, the value of $m_{1}$ either decreases by 1 or remains the same or increases by 1 .

So, as in [20], let us consider the sequence of $k(k-1) / 2+1$ words,

$$
\begin{aligned}
X^{(0)} & \stackrel{\text { def }}{=} X \\
X^{(1)} & \stackrel{\text { def }}{=}\left(X^{(0)}\right)^{(1,2)}, \\
X^{(2)} & \stackrel{\text { def }}{=}\left(X^{(1)}\right)^{(2,3)}, \\
& \vdots \\
X^{(k-1)} & \stackrel{\text { def }}{=}\left(X^{(k-2)}\right)^{(k-1, k)}, \\
X^{(k)} & \stackrel{\text { def }}{=}\left(X^{(k-1)}\right)^{(k-2, k-1)}, \\
X^{(k+1)} & \stackrel{\text { def }}{=}\left(X^{(k)}\right)^{(k-3, k-2)}, \\
& \vdots \\
X^{(2 k-3)} & \stackrel{\text { def }}{=}\left(X^{(2 k-4)}\right)^{(1,2)} \\
X^{(2 k-2)} & \stackrel{\text { def }}{=}\left(X^{(2 k-3)}\right)^{(2,3)}, \\
X^{(2 k-1)} & \stackrel{\text { def }}{=}\left(X^{(2 k-2)}\right)^{(3,4)},
\end{aligned}
$$

$\vdots$

$X^{(3 k-6)} \stackrel{\text { def }}{=}\left(X^{(3 k-7)}\right)^{(k-2, k-1)}$,

$X^{(3 k-5)} \stackrel{\text { def }}{=}\left(X^{(3 k-6)}\right)^{(k-3, k-2)}$,

$X^{(3 k-4)} \stackrel{\text { def }}{=}\left(X^{(3 k-5)}\right)^{(k-4, k-3)}$,

$\vdots$

$X^{(4 k-10)} \stackrel{\text { def }}{=}\left(X^{(4 k-9)}\right)^{(2,3)}=\left(X^{(1, k)}\right)^{(2, k-1)}$,

$\vdots$

$X^{(k(k-1) / 2)} \stackrel{\text { def }}{=}\left(X^{(k(k-1) / 2)-1}\right)^{(k / 2, k / 2+1)}=X^{R}$.

Example 1 (Word Sequence for $k=4$ ): For example, if $k=4$ and $X=x_{1} x_{2} x_{3} x_{4}$ then the sequnece has cardinality 


$$
\begin{aligned}
k(k-1) / 2+1 & =7 \text { and } \\
X^{(0)} & =x_{1} x_{2} x_{3} x_{4}=X, \\
X^{(1)} & =\underline{x_{2} x_{1} x_{3} x_{4},} \\
X^{(2)} & =x_{2} \underline{x_{3} x_{1}} x_{4}, \\
X^{(3)} & =x_{2} x_{3} \underline{x_{4}} \underline{x_{1}}, \\
X^{(4)} & =x_{2} \underline{x_{4} x_{3} x_{1}}, \\
X^{(5)} & =\underline{x_{4}} \underline{x_{2}} x_{3} \underline{x_{1}} \\
X^{(6)} & =\underline{x_{4} x_{3} x_{2} x_{1}}=X^{(1,4)},=\left(X^{(1,4)}\right)^{(2,3)}=X^{R} .
\end{aligned}
$$

The above sequence $\left\{X^{(i)}\right\}_{i=0, \ldots, k(k-1) / 2}$ is defined by a particular sequence of exchanging of consecutive bits of $X$, with $X^{(0)}=X$ and $X^{(k(k-1) / 2)}=X^{R}$. Since

$$
m_{1}\left(X^{(i+1)}\right)-m_{1}\left(X^{(i)}\right) \in\{-1,0,+1\}
$$

for all integer $i \in[0, k(k-1) / 2]$, it follows that the sequence

$$
\left\{\left(i, m_{1}\left(X^{(i)}\right)\right): i=0,1, \ldots, \frac{k(k-1)}{2}\right\}
$$

represents a "random walk" from the point $\left(0, m_{1}(X)\right)$ to the point $\left(k(k-1) / 2, m_{1}\left(X^{R}\right)\right)$ and satisfies the following key properties.

For all $X \in S(k, k / 2)$,

there exists an integer

$$
i_{s b} \in[0, k(k-1) / 2+[(k / 2) \bmod 2])
$$

such that

$$
\begin{aligned}
m_{1}\left(X^{\left(i_{s b}\right)}\right)=\left\lfloor\frac{k(k+1)}{4}\right\rfloor \\
\left(\text { or } m_{1}\left(X^{\left(i_{s b}\right)}\right)=\left\lceil\frac{k(k+1)}{4}\right\rceil\right) .
\end{aligned}
$$

In the following, we may refer to such an integer $i_{s b}$ as a "simple $m_{0}$-balancing index" for the given $m_{0}$-balanced word $X$;

and

For all $X \in S(k, k / 2)$ and

for all integers $i_{1}, i_{2} \in[0, k(k-1) / 2]$,

$$
\begin{array}{r}
m_{1}\left(X^{\left(i_{2}\right)}\right) \in\left[m_{1}\left(X^{\left(i_{1}\right)}\right)-\left|i_{2}-i_{1}\right|,\right. \\
\left.m_{1}\left(X^{\left(i_{1}\right)}\right)+\left|i_{2}-i_{1}\right|\right] .
\end{array}
$$

In [20], a simple coding scheme for 2-OSN codes is given by defining the balancing functions as $X \rightarrow X^{(i)}$, where $i \in[0, k(k-1) / 2)$. In particular, given an $m_{0}$-balanced data word $X \in S(k, k / 2)$, with $k \in 4 \mathbf{I N}$, the codeword associated with $X$ is recursively defined as

$\mathcal{E}(X)=X^{\left(i_{s b}\right)} \mathcal{E}$ (binary representation of $i_{s b}$ given in (4)).

Now we come to the definition of the set $\mathcal{C S}$ satisfying 1) of Definition 1. Let $p \in \mathbb{I N}$ and consider the partition $\left\{\Gamma_{h}\right\}_{h=0,1, \ldots, p-1}$ of a subset of $S(r, r / 2)$ into non-empty sets defined as follows. The set $\Gamma_{0}$ contains exactly one word of the set $S\left(r, r / 2, \mu_{1}\right)$ for each possible value $\mu_{1}$ in the " $m_{1}$-image" of $S(r, r / 2)$ defined to be

$$
m_{1}(S(r, r / 2)) \stackrel{\text { def }}{=}\left\{m_{1}(C): C \in S(r, r / 2)\right\} .
$$

Then let $\Gamma_{1}$ contain exactly one word of the set $S\left(r, r / 2, \mu_{1}\right)-\Gamma_{0}$ for each possible value $\mu_{1} \in m_{1}$ $\left(S(r, r / 2)-\Gamma_{0}\right)$. In general, the check word partition $\mathcal{C S}$ is defined by the following simple constructive rule

For $h=0,1,2, \ldots, p-1$,

for all integer

$$
\mu_{1} \in m_{1}\left(S(r, r / 2)-\bigcup_{j=0}^{h-1} \Gamma_{j}\right),
$$

the set $\Gamma_{h}$ contains exactly one check word in $S\left(r, r / 2, \mu_{1}\right)-\bigcup_{j=0}^{h-1} \Gamma_{j}$.

Note that such definition is well defined and implies

$$
p \leq \max _{\mu}|S(r, r / 2, \mu)| .
$$

Example 2 (Case $k=6, r=6$ and $p=3$ ): For example, if $r=6$ and $p=\max _{\mu}|S(6,3, \mu)|=3$ then

$$
\begin{aligned}
S\left(r=6, r / 2=3, \mu_{1}=6\right) & =\{111000\}, \\
S\left(r=6, r / 2=3, \mu_{1}=7\right) & =\{110100\}, \\
S\left(r=6, r / 2=3, \mu_{1}=8\right) & =\{101100,110010\}, \\
S\left(r=6, r / 2=3, \mu_{1}=9\right) & =\{011100,101010,110001\}, \\
S\left(r=6, r / 2=3, \mu_{1}=10\right) & =\{011010,100110,101001\}, \\
S\left(r=6, r / 2=3, \mu_{1}=11\right) & =\{010110,011001,100101\}, \\
S\left(r=6, r / 2=3, \mu_{1}=12\right) & =\{001110,010101,100011\}, \\
S\left(r=6, r / 2=3, \mu_{1}=13\right) & =\{001101,010011\}, \\
S\left(r=6, r / 2=3, \mu_{1}=14\right) & =\{001011\}, \\
S\left(r=6, r / 2=3, \mu_{1}=15\right) & =\{000111\} ;
\end{aligned}
$$

so that, for example, $\mathcal{C S}=\left\{\Gamma_{0}, \Gamma_{1}, \Gamma_{2}\right\}$ where

$$
\begin{aligned}
& \Gamma_{0}=\left\{\begin{array}{l}
111000, \\
110100, \\
101100, \\
011100, \\
011010, \\
010110, \\
001110, \\
001101, \\
001011, \\
000111
\end{array}\right\}, \quad \Gamma_{1}=\left\{\begin{array}{l}
110010, \\
101010, \\
100110, \\
011001, \\
010101, \\
010011
\end{array}\right\}, \\
& \Gamma_{2}=\left\{\begin{array}{l}
110001, \\
101001, \\
100101, \\
100011
\end{array}\right\} .
\end{aligned}
$$

Here, the combinatorial problem is very similar to the coding problem for balanced codes solved with the parallel decoding techniques given in [1] and [19]. In fact, the cardinalities of the constant $m_{1}$-weight codes contained in $S\left(r, \mu_{0}\right)$ show a "bell shape behaviour" similar to the binomial distribution as stated in Theorem 3 in the Appendix. In our case, if $\mathcal{C S}=\left\{\Gamma_{h}\right\}_{h \in[0, p-1]}$ is defined by (6) then Theorem 3 for $\mu_{0}=r / 2$ implies that for all integer $h \in[0, p-1]$, the statements in the following observations hold. 
Observation 4: The $m_{1}$-image of the words in $\Gamma_{h}, m_{1}\left(\Gamma_{h}\right)$, is an integer interval, say

$$
m_{1}\left(\Gamma_{h}\right) \stackrel{\text { def }}{=}\left[\alpha_{h}, \beta_{h}\right] .
$$

Observation 5: If $h>0$ then

$$
m_{1}\left(\Gamma_{h}\right)=\left[\alpha_{h}, \beta_{h}\right] \subseteq\left[\alpha_{h-1}, \beta_{h-1}\right]=m_{1}\left(\Gamma_{h-1}\right) .
$$

Observation 6: If $\alpha \stackrel{\text { def }}{=} \min _{C \in S(r, r / 2)} m_{1}(C)$ and $\beta \stackrel{\text { def }}{=}$ $\max _{C \in S(r, r / 2)} m_{1}(C)$ then the interval $m_{1}\left(\Gamma_{h}\right)=\left[\alpha_{h}, \beta_{h}\right]$ is symmetric with respect to

$$
\mu_{\text {mean }}=\frac{\alpha+\beta}{2}=\frac{\alpha_{h}+\beta_{h}}{2}=\frac{r(r+1)}{4} \in \mathbb{R},
$$

has a width of $\left(\beta_{h}-\alpha_{h}\right)=\left|\Gamma_{h}\right|-1$ where

$\left|\Gamma_{h}\right|-1 \equiv \beta_{h}-\alpha_{h} \equiv\left|\Gamma_{0}\right|-1 \equiv \beta-\alpha \equiv\left(\frac{r}{2}\right)^{2} \equiv\left(\frac{r}{2}\right) \bmod 2$.

Hence,

$$
\begin{aligned}
m_{1}\left(\Gamma_{h}\right)=\left[\alpha_{h}, \beta_{h}\right] \\
=\left[\frac{\alpha_{h}+\beta_{h}}{2}-\frac{\beta_{h}-\alpha_{h}}{2}, \frac{\alpha_{h}+\beta_{h}}{2}+\frac{\beta_{h}-\alpha_{h}}{2}\right] \\
=\left[\frac{r(r+1)}{4}-\frac{\left|\Gamma_{h}\right|-1}{2}, \frac{r(r+1)}{4}+\frac{\left|\Gamma_{h}\right|-1}{2}\right] \\
\left.=\left[\mid \frac{r(r+1)}{4}\right]-\mid \frac{\left|\Gamma_{h}\right|-1}{2}\right\rfloor, \\
{\left[\frac{r(r+1)}{4} \mid+\left\lfloor\frac{\left|\Gamma_{h}\right|-1}{2}\right\rfloor\right] . }
\end{aligned}
$$

Observation 7: Let $r \in \mathbf{I N}$ and, for all integer $\mu_{0}, \mu_{1} \in \mathbf{I N}$,

$$
s\left(r, \mu_{0}, \mu_{1}\right) \stackrel{\text { def }}{=}\left|S\left(r, \mu_{0}, \mu_{1}\right)\right| .
$$

The number of $m_{1}$-balancing functions is,

$$
\begin{aligned}
p \leq \max _{\mu \in[\alpha, \beta]}|S(r, r / 2, \mu)| & =s\left(r, r / 2,\left\lfloor\frac{r(r+1)}{4}\right\rfloor\right) \\
& =s\left(r, r / 2,\left\lceil\frac{r(r+1)}{4}\right\rceil\right) .
\end{aligned}
$$

Finally, given the above partition

$$
\mathcal{C S}=\left\{\Gamma_{h}: h \in[0, p-1]\right\},
$$

we come to the definition of each function $\left\langle\Gamma_{h}\right\rangle \in \mathcal{B}$. This is done as in [1], [12], and [19]. Namely, define the following $p$ natural numbers,

$$
\begin{aligned}
d_{h} \stackrel{\text { def }}{=} \begin{cases}0 & \text { if } h=0, \\
d_{h-1}+\left\lfloor\frac{\left|\Gamma_{h-1}\right|}{2}\right\rfloor+\left\lceil\frac{\left|\Gamma_{h}\right|}{2}\right\rceil & \text { if } h \in[1, p-1],\end{cases} \\
= \begin{cases}0 & \text { if } h=0, \\
\left\lfloor\frac{\left|\Gamma_{0}\right|}{2}\right\rfloor+\sum_{j=1}^{h-1}\left|\Gamma_{j}\right|+\left\lceil\frac{\left|\Gamma_{h}\right|}{2}\right] & \text { if } h \in[1, p-1] ;\end{cases}
\end{aligned}
$$

their associated integer intervals,

$$
I_{h} \stackrel{\text { def }}{=}\left[d_{h}-\left\lceil\frac{\left|\Gamma_{h}\right|}{2}\right\rceil+1, d_{h}+\left\lfloor\frac{\left|\Gamma_{h}\right|}{2}\right\rfloor\right],
$$

for all integer $h \in[0, p-1]$; and, also let,

$$
\begin{aligned}
I_{p} & \stackrel{\text { def }}{=}\left[d_{p-1}+\left\lfloor\frac{\left|\Gamma_{p-1}\right|}{2}\right\rfloor+1, \sum_{h=0}^{p-1}\left|\Gamma_{h}\right|\right) \\
& =\left[\left\lfloor\frac{\left|\Gamma_{0}\right|}{2}\right\rfloor+\sum_{h=1}^{p-1}\left|\Gamma_{h}\right|+1, \sum_{h=0}^{p-1}\left|\Gamma_{h}\right|\right) .
\end{aligned}
$$

Note that, from the construction (10),

1) each interval is centered in $d_{h}+\left[\left(\left|\Gamma_{h}\right|-1\right) \bmod 2\right] /$ $2 \in \mathbb{R}$, for all $h \in[0, p-1]$; and

2) the family $\left\{I_{h}: h \in[0, p]\right\}$ partitions the necessary range of the random walk index $i$, which, from (4), is given by the integer interval $[0, k(k-1) / 2+[(k / 2) \bmod 2])$. This, if $k(k-1) / 2+[(k / 2) \bmod 2] \leq \sum_{h=0}^{p-1}\left|\Gamma_{h}\right|$.

Now, given the $d_{h}$, define the associated balancing functions

$$
\begin{aligned}
& \left\langle\Gamma_{h}\right\rangle: S(k, k / 2) \rightarrow S(k, k / 2) \text { as } \\
& \left\langle\Gamma_{h}\right\rangle(X) \stackrel{\text { def }}{=} X^{\left(d_{h}\right)}, \quad \forall h \in[0, p-1] .
\end{aligned}
$$

Example 3 [Case $k=6, r=6$ and $p=3$ (Continued)]: In (7), where $r=6$ and $p=3$, from (7), (10) and (12), we have

$$
\begin{aligned}
& d_{0}=0, \\
& d_{1}=d_{0}+\left|\Gamma_{0}\right| / 2+\left|\Gamma_{1}\right| / 2=0+5+3=8 \text { and } \\
& d_{2}=d_{1}+\left|\Gamma_{1}\right| / 2+\left|\Gamma_{2}\right| / 2=8+3+2=13 .
\end{aligned}
$$

So, if $k=6$ then

$$
\begin{aligned}
& I_{0} \stackrel{\text { def }}{=}[-4,5]=\{-4,-3,-2,-1, \mathbf{0}, 1,2,3,4,5\}, \\
& I_{1} \stackrel{\text { def }}{=}[6,11]=\{6,7, \mathbf{8}, 9,10,11\}, \\
& I_{2} \stackrel{\text { def }}{=}[12,15]=\{12, \mathbf{1 3}, 14,15\}, \\
& I_{3} \stackrel{\text { def }}{=}[16,20)=\{16,17,18,19\},
\end{aligned}
$$

the random walk index

$i \in[0,15]=[0,5] \cup[6,11] \cup[12,15] \subseteq I_{0} \cup I_{1} \cup I_{2} \cup I_{3}$,

and

$$
\begin{aligned}
& \left\langle\Gamma_{0}\right\rangle(X) \stackrel{\text { def }}{=} x_{1} x_{2} x_{3} x_{4} x_{5} x_{6}=X^{(\mathbf{0})}, \\
& \left\langle\Gamma_{1}\right\rangle(X) \stackrel{\text { def }}{=} x_{2} x_{6} x_{3} x_{4} x_{5} \underline{x_{1}}=X^{(\mathbf{8})}, \\
& \left\langle\Gamma_{2}\right\rangle(X) \stackrel{\text { def }}{=} x_{6} x_{3} x_{5} x_{4} x_{2} x_{1}=X^{(\mathbf{1 3})} .
\end{aligned}
$$

We need to show that the set $\mathcal{B}$ just defined is indeed a well defined set of $m_{1}$-balancing functions. This is done in Theorem 2 below. First, note that to obtain the codewords a check word must be concatenated to some $Y=\left\langle\Gamma_{h_{b a l}}\right\rangle(X)$. So, the following theorem concerning the moments of the concatenation of words holds.

Theorem 1: For all $Y \in \mathbf{Z}_{2}^{k}$ and $C \in \mathbf{Z}_{2}^{r}$, the following relations hold.

$$
m_{0}(Y C)=m_{0}(Y)+m_{0}(C)
$$

and

$$
m_{1}(Y C)=m_{1}(Y)+m_{1}(C)+k m_{0}(C) .
$$


Proof: With regard to (14), if $Y=y_{1} y_{2} \ldots y_{k} \in \mathbf{Z}_{2}^{k}$ and $C=c_{1} c_{2} \ldots c_{r} \in \mathbf{Z}_{2}^{r}$ then

$$
\begin{aligned}
m_{1}(Y C) & =\sum_{j=1}^{k} y_{j} j+\sum_{j=k+1}^{k+r} c_{j-k} j \\
& =m_{1}(Y)+\sum_{j=1}^{r} c_{j}(k+j) \\
& =m_{1}(Y)+k \sum_{j=1}^{r} c_{j}+\sum_{j=1}^{r} c_{j} j \\
& =m_{1}(Y)+k m_{0}(C)+m_{1}(C) .
\end{aligned}
$$

Also, we need the following definition.

Definition 2 ( $m_{1}$-Crossing Index): Let $k, r \in 2 \mathbf{I N}$ and $n \stackrel{\text { def }}{=} k+r \in 4 \mathbf{I N}$. An index

$$
i_{c x} \in\left[0, \frac{k(k-1)}{2}+\left[\left(\frac{k}{2}\right) \bmod 2\right]\right)
$$

is an " $m_{1}$-crossing index" for $X \in S(k, k / 2)$ if, and only if, " $i_{c x}$ defines a point where the random walk

$$
\begin{array}{r}
R W(X) \stackrel{\text { def }}{=}\left\{\left(i, m_{1}\left(X^{(i)}\right)\right): i=0,1, \ldots, \frac{k(k-1)}{2}\right. \\
\left.+\left[\left(\frac{k}{2}\right) \bmod 2\right]-1\right\}
\end{array}
$$

crosses the $m_{1}$ middle value of $k(k+1) / 4 \in \mathbb{R}$ " - more precisely, if, and only if, one of the following two conditions hold.

1) $k, r \in 4 \mathbf{I N}$ and $i_{c x}$ is a simple $m_{1}$-balancing index for $X$ according to (4); that is,

$$
m_{1}\left(X^{\left(i_{c x}\right)}\right)=\frac{k(k+1)}{4} \in \mathbf{I N} ;
$$

2) $k, r \in 4 \mathbf{I N}+2$ and $i_{c x}$ is a simple decreasing $m_{1}$-balancing index for $X$; that is,

$$
\begin{aligned}
m_{1}\left(X^{\left(i_{c x}-1\right)}\right) & =\left\lceil\frac{k(k+1)}{4}\right\rceil \\
& >\left\lfloor\frac{k(k+1)}{4}\right\rfloor=m_{1}\left(X^{\left(i_{c x}\right)}\right) .
\end{aligned}
$$

3) $k, r \in 4 \mathbf{I N}+2$ and $i_{c x}$ is a simple increasing $m_{1}$-balancing index for $X$; that is,

$$
\begin{aligned}
m_{1}\left(X^{\left(i_{c x}-1\right)}\right) & =\left\lfloor\frac{k(k+1)}{4}\right\rfloor \\
& <\left\lceil\frac{k(k+1)}{4}\right\rceil=m_{1}\left(X^{\left(i_{c x}\right)}\right) .
\end{aligned}
$$

We note that, for all $X \in S(k, k / 2)$ there always exists at least one $m_{1}$-crossing index, $i_{c x}$, of $X$ because of Observation 2 and Observation 3. If $k, r \in 4 \mathbf{I N}$ then $i_{c x}$ coincides with the index $i_{s b}$ in (4). If $k, r \in 4 \mathbf{I N}+2$ and $m_{1}(X)<k(k+1) / 4$ then there exist at least an index $i_{c x}$ such that (17) holds. If instead, $k, r \in 4 \mathbf{I N}+2$ and $m_{1}(X)>k(k+1) / 4$ then there exist an index $i_{c x}$ such that (16) holds. Also, note that if $k, r \in 4 \mathbf{I N}+2$ then the definition of $m_{1}$-crossing index is stronger than the definition of simple $m_{1}$-balancing index as the following example shows.

Example 4 [Case $k=6, r=6$ and $p=3$ (Continued)]: If $k=6 \in 4 \mathbb{N}+2$ then $10=\lfloor k(k+1) / 4\rfloor<$ $\lfloor k(k+1) / 4\rfloor=11$. The random walk sequence of the word $X=010011 \in S(6,3)$ is

$$
\begin{aligned}
& X^{\left(d_{0}\right)}=X^{(\mathbf{0})}=010011, \quad m_{1}\left(X^{(\mathbf{0})}\right)=13, \\
& X^{(1)}=\underline{100011,} m_{1}\left(X^{(1)}\right)=12 \text {, } \\
& X^{(2)}=1 \underline{00011}, m_{1}\left(X^{(2)}\right)=12 \text {, } \\
& X^{(3)}=10 \underline{0011}, m_{1}\left(X^{(3)}\right)=12 \text {, } \\
& X^{(4)}=100 \underline{101}, \quad m_{1}\left(X^{(4)}\right)=11 \text {, } \\
& X^{(5)}=1001 \underline{10}, \quad m_{1}\left(X^{(5)}\right)=10, \longleftarrow \\
& X^{(6)}=100 \underline{110}, \quad m_{1}\left(X^{(6)}\right)=10 \text {, } \\
& X^{(7)}=10 \underline{10} \underline{10}, \quad m_{1}\left(X^{(7)}\right)=9 \text {, } \\
& X^{\left(d_{1}\right)}=X^{(\mathbf{8})}=1 \underline{1001} \underline{0}, \quad m_{1}\left(X^{(\mathbf{1 5})}\right)=8 \text {, } \\
& X^{(9)}=\underline{110010}, \quad m_{1}\left(X^{(9)}\right)=8 \text {, } \\
& X^{(10)}=\underline{10101} \underline{0}, \quad m_{1}\left(X^{(10)}\right)=9 \text {, } \\
& X^{(11)}=\underline{1001} \underline{0}, \quad m_{1}\left(X^{(11)}\right)=10, \\
& X^{(12)}=\underline{100110}, m_{1}\left(X^{(12)}\right)=10, \\
& X^{\left(d_{2}\right)}=X^{(\mathbf{1 3})}=\underline{101010}, \quad m_{1}\left(X^{(\mathbf{1 5})}\right)=9 \text {, } \\
& X^{(14)}=\underline{110010}, \quad m_{1}\left(X^{(14)}\right)=8 \text {, } \\
& X^{(15)}=\underline{110010}, m_{1}\left(X^{(13)}\right)=8 \text {. }
\end{aligned}
$$

Hence, $X$ has only one $m_{1}$-crossing index $i_{c x}=5$ (indicated by the arrow to the right) because (16) holds. In particular, $i_{c x}=5$ is a decreasing $m_{1}$-crossing index for the random walk $R W(X)$.

Now we come to the main general theorem.

Theorem 2: Let $k, r \in 2 \mathbf{I N}$ be given so that $n \stackrel{\text { def }}{=} k+r \in$ $4 \mathbf{I N}$. Let $p \in \mathbf{I N}$,

$$
\mathcal{C S}=\left\{\Gamma_{0}, \Gamma_{1}, \ldots, \Gamma_{p-1}\right\}
$$

be a partition of a subset of $S(r, r / 2)$ such that (8) holds for all $h \in[0, p-1]$ and let

$$
\mathcal{B}=\left\{\left\langle\Gamma_{h}\right\rangle: h \in[0, p-1]\right\}
$$

be the set of functions defined by (10) and (12). If

$$
\begin{aligned}
& \frac{k(k-1)}{2} \leq \sum_{h=0}^{p-1}\left|\Gamma_{h}\right|-\epsilon(k, r), \text { with } \\
& \epsilon(k, r) \stackrel{\text { def }}{=} \begin{cases}0 & \text { if } k, r \in 4 \mathbf{I N}, \\
1 & \text { otherwise, }\end{cases}
\end{aligned}
$$


then $\mathcal{B}$ is a set of $m_{1}$-balancing functions according to Definition 1. In particular, if we let

$$
\begin{aligned}
p & =\left|S\left(r, r / 2,\left\lfloor\frac{r(r+1)}{4}\right\rfloor\right)\right|, \\
\bigcup_{h=0}^{p-1}\left|\Gamma_{h}\right| & =S(r, r / 2)
\end{aligned}
$$

and

$$
\frac{k(k-1)}{2} \leq\left(\begin{array}{c}
r \\
r / 2
\end{array}\right)
$$

then $\mathcal{B}$ is a set of $m_{1}$-balancing functions. Note that the inequality in (19) is the leftmost relation in (2).

Proof: First note that

$$
\begin{aligned}
& \left\lceil\frac{k(k+1)}{4}\right\rceil+\left\lfloor\frac{r(r+1)}{4}\right\rfloor+\frac{k r}{2} \\
& \quad=\left\lfloor\frac{k(k+1)}{4}\right\rfloor+\left\lceil\frac{r(r+1)}{4}\right\rceil+\frac{k r}{2} \\
& \quad=\frac{n(n+1)}{4}
\end{aligned}
$$

because $k, r \in 2 \mathbf{I N}$ and $n \in 4 \mathbf{I N}$.

Property 1 ) of Definition 1 follows because the family $\mathcal{C S}$ is a partition. With regard to the first moment relation in property 2) of Definition 1, given any $X \in S(k, k / 2)$, we need to find/define an $m_{1}$-balancing index, $\hat{h} \in h_{\text {bal }}(X)$, for the proposed refined coding scheme in each of the cases which follow.

Case $k, r \in 4 \mathbf{I N}$. Let $i_{c x}$ be an $m_{1}$-crossing index of $X$. For such index,

$$
i_{c x} \in\left[0, \frac{k(k-1)}{2}\right) \text { and } m_{1}\left(X^{\left(i_{c x}\right)}\right)=\frac{k(k+1)}{4} \in \mathbf{I N}
$$

hold because of Definition 2. In this case, the integer intervals in (11) are given by

$$
I_{h} \stackrel{\text { def }}{=}\left[d_{h}-\frac{\left|\Gamma_{h}\right|-1}{2}, d_{h}+\frac{\left|\Gamma_{h}\right|-1}{2}\right],
$$

for all integer $h \in[0, p-1]$; and,

$$
I_{p} \stackrel{\text { def }}{=}\left[\left\lfloor\frac{\left|\Gamma_{0}\right|}{2}\right\rfloor+\sum_{h=1}^{p-1}\left|\Gamma_{h}\right|+1, \sum_{h=0}^{p-1}\left|\Gamma_{h}\right|\right) .
$$

From (18), the family $\left\{I_{h}: h \in[0, p]\right\}$ partitions the index range interval $[0, k(k-1) / 2)$. So, there exists an integer $h \in[0, p]$ such that $i_{c x} \in I_{h}$. Exactly one of the following subcases must hold.

A1: $h \in[0, p-1]$. Here, from (22),

$$
\delta i \stackrel{\text { def }}{=} i_{c x}-d_{h} \in\left[-\frac{\left|\Gamma_{h}\right|-1}{2}, \frac{\left|\Gamma_{h}\right|-1}{2}\right] .
$$

So, from (5),

$$
\begin{aligned}
m_{1}\left(X^{\left(d_{h}\right)}\right) & \in\left[m_{1}\left(X^{\left(i_{c x}\right)}\right)-|\delta i|, m_{1}\left(X^{\left(i_{c x}\right)}\right)+|\delta i|\right] \\
& =\left[\frac{k(k+1)}{4}-|\delta i|, \frac{k(k+1)}{4}+|\delta i|\right] \\
& \subseteq\left[\frac{k(k+1)}{4}-\frac{\left|\Gamma_{h}\right|-1}{2}, \frac{k(k+1)}{4}+\frac{\left|\Gamma_{h}\right|-1}{2}\right] ;
\end{aligned}
$$

and so, there always exists a "small $m_{1}$-imbalance"

$$
\mu \in[-|\delta i|,|\delta i|] \cap \mathbf{N} \subseteq\left[-\frac{\left|\Gamma_{h}\right|-1}{2}, \frac{\left|\Gamma_{h}\right|-1}{2}\right]
$$

such that,

$$
\begin{aligned}
& m_{1}\left(X^{\left(d_{h}\right)}\right)=\frac{k(k+1)}{4}-\mu \in\left[\frac{k(k+1)}{4}-\frac{\left|\Gamma_{h}\right|-1}{2},\right. \\
& \left.\frac{k(k+1)}{4}+\frac{\left|\Gamma_{h}\right|-1}{2}\right] .
\end{aligned}
$$

Hence, from (8), there exists $C \in \Gamma_{h}$ with

$$
\begin{aligned}
m_{1}(C)= & \frac{r(r+1)}{4} \\
& +\mu \in\left[\frac{r(r+1)}{4}-\frac{\left|\Gamma_{h}\right|-1}{2}, \frac{r(r+1)}{4}+\frac{\left|\Gamma_{h}\right|-1}{2}\right] \\
= & m_{1}\left(\Gamma_{h}\right)
\end{aligned}
$$

such that

$$
\begin{aligned}
m_{1}\left(\left\langle\Gamma_{h}\right\rangle(X) C\right) & =m_{1}\left(X^{\left(d_{h}\right)}\right)+m_{1}(C)+k m_{0}(C) \\
& =\left(\frac{k(k+1)}{4}-\mu\right)+\left(\frac{r(r+1)}{4}+\mu\right)+\frac{k r}{2} \\
& =\frac{n(n+1)}{4},
\end{aligned}
$$

because of (14) in Theorem 1 with

$$
\begin{aligned}
m_{1}(Y) & =m_{1}\left(X^{\left(d_{h}\right)}\right)=k(k+1) / 4-\mu, \\
m_{1}(C) & =r(r+1) / 4+\mu \text { and } \\
k m_{0}(C) & =k r / 2 ;
\end{aligned}
$$

and also because of (20). This means that $\hat{h} \stackrel{\text { def }}{=} h \in h_{b a l}(X)$ is a balancing index for this refined coding scheme.

A2: $h=p$. In this case, from (22) and Definition 2,

$$
i_{c x} \in\left[\left\lfloor\frac{\left|\Gamma_{0}\right|}{2}\right\rfloor+\sum_{h=1}^{p-1}\left|\Gamma_{h}\right|+1, \frac{k(k-1)}{2}\right) .
$$

So, from (18),

$$
\begin{aligned}
\frac{k(k-1)}{2}-i_{c x} & \in\left[1, \frac{k(k-1)}{2}-\left\lfloor\frac{\left|\Gamma_{0}\right|}{2}\right\rfloor-\sum_{h=1}^{p-1}\left|\Gamma_{h}\right|\right) \\
& \subseteq\left[1,\left\lceil\frac{\left|\Gamma_{0}\right|}{2}\right\rceil\right) .
\end{aligned}
$$

Hence, from (5), (21) and the above relation,

$$
\begin{aligned}
m_{1}\left(X^{R}\right)= & m_{1}\left(X^{(k(k-1) / 2)}\right) \\
\in & {\left[m_{1}\left(X^{\left(i_{c x}\right)}\right)-\left(\frac{k(k-1)}{2}-i_{c x}\right),\right.} \\
& \left.m_{1}\left(X^{\left(i_{c x}\right)}\right)+\left(\frac{k(k-1)}{2}-i_{c x}\right)\right] \\
\subseteq & {\left[\frac{k(k+1)}{4}-\left(\left\lceil\frac{\left|\Gamma_{0}\right|}{2}\right]-1\right),\right.} \\
& \frac{k(k+1)}{4}+\left(\left[\frac{\left|\Gamma_{0}\right|}{2} \mid-1\right)\right] \\
= & {\left[\frac{k(k+1)}{4}-\frac{\left|\Gamma_{0}\right|-1}{2}, \frac{k(k+1)}{4}+\frac{\left|\Gamma_{0}\right|-1}{2}\right] . }
\end{aligned}
$$


This, from Observation 2, implies,

$$
\begin{aligned}
m_{1}(X)=\frac{k(k+1)}{2}-m_{1}\left(X^{R}\right) \in & {\left[\frac{k(k+1)}{4}-\frac{\left|\Gamma_{0}\right|-1}{2},\right.} \\
& \left.\frac{k(k+1)}{4}+\frac{\left|\Gamma_{0}\right|-1}{2}\right] ;
\end{aligned}
$$

and so, there exists a "small $m_{1}$-imbalance" $\mu \in \mathbf{I N}$ such that,

$$
\begin{aligned}
m_{1}(X)=\frac{k(k+1)}{4}-\mu \in\left[\frac{k(k+1)}{4}-\frac{\left|\Gamma_{0}\right|-1}{2},\right. \\
\left.\frac{k(k+1)}{4}+\frac{\left|\Gamma_{0}\right|-1}{2}\right] .
\end{aligned}
$$

Again, from (8), there exists $C \in \Gamma_{0}$ with

$$
\begin{aligned}
m_{1}(C)= & \frac{r(r+1)}{4} \\
& +\mu \in\left[\frac{r(r+1)}{4}-\frac{\left|\Gamma_{0}\right|-1}{2}, \frac{r(r+1)}{4}+\frac{\left|\Gamma_{0}\right|-1}{2}\right] \\
= & m_{1}\left(\Gamma_{0}\right)
\end{aligned}
$$

such that $m_{1}\left(\left\langle\Gamma_{0}\right\rangle(X) C\right)=n(n+1) / 4$ because of Theorem 1 and (20). So, if $i_{c x} \in I_{p}$ then $\hat{h} \stackrel{\text { def }}{=} 0 \in h_{b a l}(X)$ is a balancing index for the refined coding scheme.

Case $k, r \in 4 \mathbf{I N}+2$. Let

$$
i_{c x} \in\left[0, \frac{k(k-1)}{2}\right]=\left[0, \frac{k(k-1)}{2}+1\right)
$$

be an $m_{1}$-crossing index of $X$ defined in Definition 2 . Here, the integer intervals in (11) are given by

$$
I_{h} \stackrel{\text { def }}{=}\left[d_{h}-\frac{\left|\Gamma_{h}\right|}{2}+1, d_{h}+\frac{\left|\Gamma_{h}\right|}{2}\right],
$$

for all integer $h \in[0, p-1]$; and,

$$
I_{p} \stackrel{\text { def }}{=}\left[\frac{\left|\Gamma_{0}\right|}{2}+\sum_{h=1}^{p-1}\left|\Gamma_{h}\right|+1, \sum_{h=0}^{p-1}\left|\Gamma_{h}\right|\right) .
$$

From (18) and $\epsilon(k, r)=1$, the family $\left\{I_{h}: h \in[0, p]\right\}$ partitions the index range interval $[0, k(k-1) / 2]$. So, there exists an integer $h \in[0, p]$ such that $i_{c x} \in I_{h}$. Based on (23), exactly one of the following subcases must hold.

B1: $h \in[0, p-1]$, (16) holds and

$$
\begin{aligned}
i_{c x}=d_{h}+\frac{\left|\Gamma_{h}\right|}{2} & \Longleftrightarrow \delta i \stackrel{\text { def }}{=}\left(i_{c x}-1\right)-d_{h} \\
& =\frac{\left|\Gamma_{h}\right|}{2}-1=\frac{\left|\Gamma_{h}\right|-2}{2} .
\end{aligned}
$$

In this case, from (5) and (16), there always exists a "small $m_{1}$-imbalance" $\mu \in \mathbf{I N}$ such that,

$$
\begin{aligned}
m_{1}\left(X^{\left(d_{h}\right)}\right)= & \left\lceil\frac{k(k+1)}{4}\right\rceil \\
& -\mu \in\left[m_{1}\left(X^{\left(i_{c x}-1\right)}\right)-\delta i, m_{1}\left(X^{\left(i_{c x}-1\right)}\right)+\delta i\right] \\
= & \left\lceil\left\lceil\frac{k(k+1)}{4}\right\rceil-\frac{\left|\Gamma_{h}\right|-2}{2},\right. \\
& \left.\left\lceil\frac{k(k+1)}{4}\right\rceil+\frac{\left|\Gamma_{h}\right|-2}{2}\right] .
\end{aligned}
$$

Hence, from (8), there exists $C \in \Gamma_{h}$ with

$$
\begin{aligned}
& m_{1}(C)=\left\lfloor\frac{r(r+1)}{4}\right\rfloor \\
&+\mu \in\left[\left\lfloor\frac{r(r+1)}{4}\right\rfloor-\frac{\left|\Gamma_{h}\right|-2}{2},\right. \\
&\left.\left\lfloor\frac{r(r+1)}{4}\right\rfloor+\frac{\left|\Gamma_{h}\right|-2}{2}\right\rfloor \subseteq m_{1}\left(\Gamma_{h}\right)
\end{aligned}
$$

such that

$$
\begin{aligned}
m_{1} & \left(\left\langle\Gamma_{h}\right\rangle(X) C\right) \\
& =m_{1}\left(X^{\left(d_{h}\right)}\right)+m_{1}(C)+k m_{0}(C) \\
& =\left(\left\lceil\frac{k(k+1)}{4}\right\rceil-\mu\right)+\left(\left\lfloor\frac{r(r+1)}{4}\right\rfloor+\mu\right)+\frac{k r}{2} \\
& =\frac{n(n+1)}{4}
\end{aligned}
$$

because of Theorem 1 and (20). This means that $\hat{h} \stackrel{\text { def }}{=} h \in$ $h_{b a l}(X)$ is a balancing index for the refined coding scheme.

B2: $h \in[0, p-1],(16)$ holds and

$$
\begin{aligned}
i_{c x} & \in\left[d_{h}-\left(\frac{\left|\Gamma_{h}\right|}{2}-1\right), d_{h}+\left(\frac{\left|\Gamma_{h}\right|}{2}-1\right)\right] \\
& \Longleftrightarrow \delta i \stackrel{\text { def }}{=} i_{c x}-d_{h} \in\left[-\frac{\left|\Gamma_{h}\right|-2}{2}, \frac{\left|\Gamma_{h}\right|-2}{2}\right] .
\end{aligned}
$$

In this case, from (5) and (16), there always exists a "small $m_{1}$-imbalance" $\mu \in \mathbf{I N}$ such that,

$$
\begin{aligned}
& m_{1}\left(X^{\left(d_{h}\right)}\right)=\left\lfloor\frac{k(k+1)}{4}\right\rfloor \\
& -\mu \in\left[m_{1}\left(X^{\left(i_{c x}\right)}\right)-|\delta i|, m_{1}\left(X^{\left(i_{c x}\right)}\right)+|\delta i|\right] \\
& =\left[\left\lfloor\frac{k(k+1)}{4}\right\rfloor-|\delta i|,\left\lfloor\frac{k(k+1)}{4}\right\rfloor+|\delta i|\right] \\
& \subseteq\left[\left\lfloor\frac{k(k+1)}{4}\right\rfloor-\frac{\left|\Gamma_{h}\right|-2}{2}\right. \text {, } \\
& \left.\left\lfloor\frac{k(k+1)}{4}\right\rfloor+\frac{\left|\Gamma_{h}\right|-2}{2}\right\rfloor \text {. }
\end{aligned}
$$

Hence, from (8), there exists $C \in \Gamma_{h}$ with

$$
\begin{aligned}
m_{1}(C)=\left\lceil\frac{r(r+1)}{4}\right\rceil+ & \mu \in\left[\left\lceil\frac{r(r+1)}{4}\right\rceil-\frac{\left|\Gamma_{h}\right|-2}{2},\right. \\
& {\left.\left[\frac{r(r+1)}{4}\right\rceil+\frac{\left|\Gamma_{h}\right|-2}{2}\right] \subseteq m_{1}\left(\Gamma_{h}\right) }
\end{aligned}
$$

such that

$$
\begin{aligned}
m_{1} & \left(\left\langle\Gamma_{h}\right\rangle(X) C\right) \\
& =m_{1}\left(X^{\left(d_{h}\right)}\right)+m_{1}(C)+k m_{0}(C) \\
& =\left(\left\lfloor\frac{k(k+1)}{4}\right\rfloor-\mu\right)+\left(\left\lceil\frac{r(r+1)}{4}\right\rceil+\mu\right)+\frac{k r}{2} \\
& =\frac{n(n+1)}{4}
\end{aligned}
$$

because of Theorem 1 and (20). This means that $\hat{h} \stackrel{\text { def }}{=} h \in h_{b a l}(X)$. 
B3: $h=p$, (16) holds and

$$
i_{c x} \in\left[\frac{\left|\Gamma_{0}\right|}{2}+\sum_{h=1}^{p-1}\left|\Gamma_{h}\right|+1, \frac{k(k-1)}{2}\right] .
$$

So, from (18) and $\epsilon(k, r)=1$,

$$
\begin{aligned}
\frac{k(k-1)}{2}-i_{c x} & \in\left[0, \frac{k(k-1)}{2}-\frac{\left|\Gamma_{0}\right|}{2}-\sum_{h=1}^{p-1}\left|\Gamma_{h}\right|\right] \\
& \subseteq\left[0, \frac{\left|\Gamma_{0}\right|}{2}-1\right] .
\end{aligned}
$$

Hence, from (5), (16) and the above relation,

$$
\begin{aligned}
& m_{1}\left(X^{R}\right)= m_{1}\left(X^{(k(k-1) / 2)}\right) \\
& \in {\left[m_{1}\left(X^{\left(i_{c x}\right)}\right)-\left(\frac{k(k-1)}{2}-i_{c x}\right),\right.} \\
&\left.m_{1}\left(X^{\left(i_{c x}\right)}\right)+\left(\frac{k(k-1)}{2}-i_{c x}\right)\right] \\
& \subseteq {\left[\left\lfloor\frac{k(k+1)}{4}\right\rfloor-\frac{\left|\Gamma_{0}\right|-2}{2},\right.} \\
&\left.\left\lfloor\frac{k(k+1)}{4}\right\rfloor+\frac{\left|\Gamma_{0}\right|-2}{2}\right]
\end{aligned}
$$

This, from Observation 2, implies,

$$
\begin{aligned}
m_{1}(X)=\frac{k(k+1)}{2}-m_{1}\left(X^{R}\right) \in & {\left[\left\lceil\frac{k(k+1)}{4}\right\rceil-\frac{\left|\Gamma_{0}\right|-2}{2},\right.} \\
& \left.\left\lceil\frac{k(k+1)}{4}\right\rceil+\frac{\left|\Gamma_{0}\right|-2}{2}\right] ;
\end{aligned}
$$

and so, there exists a "small $m_{1}$-imbalance" $\mu \in \mathbb{I N}$ such that,

$$
\begin{aligned}
m_{1}(X)=\left\lceil\frac{k(k+1)}{4}\right\rceil-\mu \in[ & {\left[\frac{k(k+1)}{4}\right\rceil-\frac{\left|\Gamma_{0}\right|-2}{2}, } \\
& \left.\left\lceil\frac{k(k+1)}{4}\right\rceil+\frac{\left|\Gamma_{0}\right|-2}{2}\right] .
\end{aligned}
$$

Hence, from (8), there exists $C \in \Gamma_{0}$ with

$$
\begin{aligned}
m_{1}(C)=\left\lfloor\frac{r(r+1)}{4}\right\rfloor & +\mu \in\left\lfloor\left\lfloor\frac{r(r+1)}{4}\right\rfloor-\frac{\left|\Gamma_{0}\right|-2}{2},\right. \\
& \left.\left\lfloor\frac{r(r+1)}{4}\right\rfloor+\frac{\left|\Gamma_{0}\right|-2}{2}\right\rfloor \subseteq m_{1}\left(\Gamma_{0}\right)
\end{aligned}
$$

such that $m_{1}\left(\left\langle\Gamma_{0}\right\rangle(X) C\right)=n(n+1) / 4$ because of Theorem 1 and (20). So, if $i_{c x} \in I_{p}$ then $\hat{h} \stackrel{\text { def }}{=} 0 \in h_{b a l}(X)$.

C1: $h \in[0, p-1],(17)$ holds and

$$
\begin{aligned}
i_{c x}=d_{h}+\frac{\left|\Gamma_{h}\right|}{2} & \left.\Longleftrightarrow \delta i \stackrel{\text { def }}{=}\left(i_{c x}-1\right)-d_{h}\right] \\
& =\frac{\left|\Gamma_{h}\right|}{2}-1=\frac{\left|\Gamma_{h}\right|-2}{2} .
\end{aligned}
$$

This case follows exactly as the case B1 where the floor function is exchanged with the ceiling function. Here $\hat{h} \stackrel{\text { def }}{=} h \in h_{\text {bal }}(X)$.

C2: $h \in[0, p-1],(17)$ holds and

$$
\begin{aligned}
i_{c x} \in & {\left[d_{h}-\left(\frac{\left|\Gamma_{h}\right|}{2}-1\right), d_{h}+\left(\frac{\left|\Gamma_{h}\right|}{2}-1\right)\right] } \\
& \Longleftrightarrow \delta i \stackrel{\text { def }}{=} i_{c x}-d_{h} \in\left[-\frac{\left|\Gamma_{h}\right|-2}{2}, \frac{\left|\Gamma_{h}\right|-2}{2}\right] .
\end{aligned}
$$

This case follows exactly as the case $\mathbf{B 2}$ where the floor function is exchanged with the ceiling function. Here $\hat{h} \stackrel{\text { def }}{=} h \in h_{b a l}(X)$.

C3: $h=p$, (17) holds and

$$
i_{c x} \in\left[\frac{\left|\Gamma_{0}\right|}{2}+\sum_{h=1}^{p-1}\left|\Gamma_{h}\right|+1, \frac{k(k-1)}{2}\right] .
$$

This case follows exactly as the case $\mathbf{B 3}$ where the floor function is exchanged with the ceiling function. Here $\hat{h} \stackrel{\text { def }}{=} 0 \in h_{\text {bal }}(X)$.

Since no other cases are left, property 2 ) holds. As we can see, property 2) follows similarly to the proof of [19, Th. 4]. In fact, here we have even proven a slightly stronger result; namely, we have shown that if an $m_{1}$-crossing index $i_{c x} \in[0, k(k-1) / 2+[(k / 2) \bmod 2])$ of the random walk $R W(X)$ in (15) lies in $I_{h}$ then $h \in h_{b a l}(X) \in[0, p-1]$ is a balancing index for the refined scheme. This, modulus the necessary length of the random walk given by $k(k-1) / 2+$ [( $k / 2) \bmod 2]$ (see case A2, B3 and C3 above). In Section III, we will make a good use of this improved result.

Property 3) of Definition 1 holds because the functions $\left\langle\Gamma_{h}\right\rangle$ in (12) are permutations of the components of $X$. So, $\mathcal{B}$ is a set of $m_{1}$-balancing functions according to Definition 1 . In particular, this statement holds true if we let $\bigcup_{h=0}^{p-1}\left|\Gamma_{h}\right|=$ $S(r, r / 2)$. In this case, $k$ must satisfy

$$
\frac{k(k-1)}{2} \leq|S(r, r / 2)|-\epsilon(k, r) ;
$$

which is equivalent to $k(k-1) / 2 \leq\left(\begin{array}{c}r \\ r / 2\end{array}\right)$ because $k, r \in 2 \mathbf{I N}$, $n \in 4 \mathbf{I N}$ and because whenever $k, r \in 4 \mathbf{I N}+2$ it follows $k(k-1) / 2 \in 2 \mathbf{I N}+1$ and $\left(\begin{array}{c}r \\ r / 2\end{array}\right) \in 2 \mathbf{I N}$.

At this point, the encoding of an information word is done as follows.

Algorithm 1 General Encoding Algorithm

Input: the information word $Z \in \mathbf{Z}_{2}^{\tilde{k}}$.

Output: the codeword

$$
Y C=\mathcal{E}_{2}\left(\mathcal{E}_{1}(Z)\right)=\left(\mathcal{E}_{1} \circ \mathcal{E}_{2}\right)(Z) \in \mathcal{S N}(n, 2)
$$

where $n=k+r, Y \in \mathbf{Z}_{2}^{k}$ and $C \in \mathbf{Z}_{2}^{r}$.

Perform steps S0, S1 and S2.

S0: $m_{0}$-balance the information word $Z$. For example, any $m_{0}$-balancing method given in [1], [2], [4], [7], [12], [18], [19] or [21] can be used. Let $X$ be the $m_{0}$-balanced information word of length $k$ associated with $Z$; i. e., $X=\mathcal{E}_{1}(Z)$. In this way, $m_{0}(X)=k / 2$.

S1: find a balancing index of $X$ and then compute the relative information and check part of the codeword. For the time being, assume this is done exhaustively by computing the set, $h_{b a l}(X)$, of all possible balancing indices associated with $X$ as follows. For all $h \in[0, p-1]$ do steps S1.1, S1.2, S1.3 and S1.4.

S1.1: compute $\left\langle\Gamma_{h}\right\rangle(X)=X^{\left(d_{h}\right)}$.

S1.2: compute $m_{1}\left(\left\langle\Gamma_{h}\right\rangle(X)\right)$. 
S1.3: compute the $m_{1}$-unbalance

$$
\mu_{1}=\frac{n(n+1)}{4}-m_{1}\left(\left\langle\Gamma_{h}\right\rangle(X)\right)-\frac{k r}{2} .
$$

S1.4: if there exists $C \in \Gamma_{h}$ such that $m_{1}(C)=\mu_{1}$ then a balancing index $h_{b a l} \stackrel{\text { def }}{=} h$ is found and the relative $m_{1}$-balancing check is $C_{h_{b a l}} \stackrel{\text { def }}{=} C \in \Gamma_{h_{b a l}}$. Note that such $m_{1}$-balancing check exists in $\Gamma_{h}$ if, and only if, $\mu_{1} \in m_{1}\left(\Gamma_{h}\right)=\left[\alpha_{h}, \beta_{h}\right]$ (because of Observation 4 above).

S2: for the $m_{0}$-balanced information word $X$, select the $m_{1}$ balanced codeword as

$$
\mathcal{E}_{2}(X)=Y C=\left\langle\Gamma_{h_{b a l}}\right\rangle(X) C_{h_{b a l}}=X^{\left(d_{h_{b a l}}\right)} C_{h_{b a l}} ;
$$

where $h_{b a l} \in h_{b a l}(X) \subseteq[0, p-1]$ is one among all possible balancing indices found in step $\mathrm{S} 1$. In this way, the information word $Z$ is encoded into the 2-OSN word $Y C=\mathcal{E}_{2}(X)=$ $\mathcal{E}_{2}\left(\mathcal{E}_{1}(Z)\right)$.

S3: Output $\mathcal{E}_{2}(X)$ and exit.

On the other hand, the decoding is performed as follows.

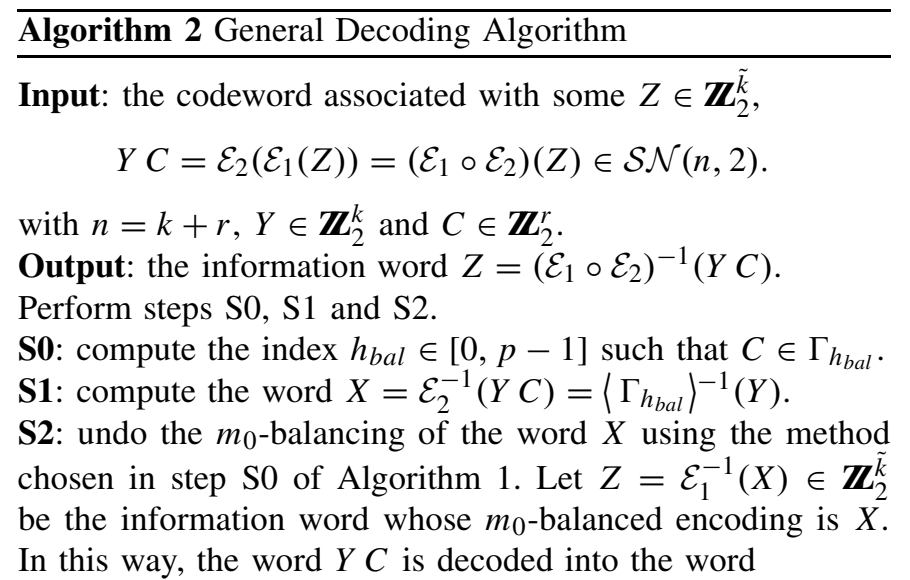

$$
Z=\mathcal{E}_{1}^{-1}(X)=\mathcal{E}_{1}^{-1}\left(\mathcal{E}_{2}^{-1}(Y C)\right)=\left(\mathcal{E}_{1} \circ \mathcal{E}_{2}\right)^{-1}(Y C)
$$

$\underline{\text { S3: Output } Z \text { and exit. }}$

The following example shows the $m_{1}$-balancing part of Algorithm 1 and the $m_{1}$-unbalancing part of Algorithm 2.

Example 5 (Code Design for $k=12, r=8$ and $p=8$ ): Let the number of $m_{0}$-balanced information bits be $k=12 \in 2 \mathbf{N N}$. So, if $r=8 \in 2 \mathbf{I N}$ then relation (19) holds because

$$
\frac{k(k-1)}{2}=66 \leq 70=\left(\begin{array}{l}
8 \\
4
\end{array}\right)=\left(\begin{array}{c}
r \\
r / 2
\end{array}\right) .
$$

This means that with $r=8$, by letting

$$
\bigcup_{h=0}^{p-1}\left|\Gamma_{h}\right|=S(8,4)
$$

a code design of length $n=k+r=20$ with

$$
p=s\left(8,4,8 \cdot \frac{9}{4}=18\right)=8
$$

$m_{1}$-balancing functions can be given to $m_{1}$-balance the $k=12$ $m_{0}$-balanced bits. Columns 2 through 8 of Table I define a particular partition $\mathcal{C S}=\left\{\Gamma_{0}, \Gamma_{1}, \ldots, \Gamma_{7}\right\}$. We readily note that $\Gamma_{7}$ is redundant and a code design can be given with $p=7 m_{1}$-balancing functions by using the first $7 \Gamma_{h}$. This is because

$$
\frac{k(k-1)}{2}=66 \leq 69=70-\left|\Gamma_{7}\right|=\sum_{h=0}^{6}\left|\Gamma_{h}\right|
$$

and so (18) is satisfied. However, note that by using $p=8$, instead of 7, $m_{1}$-balancing functions we increase the average number of possible encodings of a given information word. Thus, by applying the same method given in [12], [16], and [29] for balanced codes to the 2-OSN codes given here, a little more extra information can be conveyed by the code. Now, the $m_{0}$-weight of every codeword must be equal to $n / 2=10$ and the $m_{1}$-weight must be equal to $n(n+1) / 4=105$. Suppose

$$
X=100000101111
$$

is the given $m_{0}$-balanced word which needs to be encoded into a word of $\mathcal{S N}(20,2)$. The entire random walk sequence for $X$ is given below (where the left arrows to the right indicate a word in $\mathcal{S N}(12,2)$ ).

$$
\begin{aligned}
& X^{\left(d_{0}\right)}=X^{(\mathbf{0})}=100000101111, m_{1}\left(X^{(\mathbf{0})}\right)=50, \\
& X^{(1)}=\underline{010000101111,} m_{1}\left(X^{(1)}\right)=51 \text {, } \\
& X^{(2)}=0 \underline{01000101111,} m_{1}\left(X^{(2)}\right)=52 \text {, } \\
& X^{(3)}=0 \underline{0100101111,} m_{1}\left(X^{(3)}\right)=53 \text {, } \\
& X^{(4)}=000 \underline{010101111,} m_{1}\left(X^{(4)}\right)=54 \text {, } \\
& X^{(5)}=0000 \underline{01101111,} m_{1}\left(X^{(5)}\right)=55 \text {, } \\
& X^{(6)}=00000 \underline{1101111,} m_{1}\left(X^{(6)}\right)=55 \text {, } \\
& X^{(7)}=000001 \underline{011111}, m_{1}\left(X^{(7)}\right)=56 \text {, } \\
& X^{(8)}=0000010 \underline{11111}, m_{1}\left(X^{(8)}\right)=56 \text {, } \\
& X^{(9)}=00000101 \underline{1111}, m_{1}\left(X^{(9)}\right)=56 \text {, } \\
& X^{(10)}=000001011 \underline{111}, \quad m_{1}\left(X^{(10)}\right)=56 \text {, } \\
& X^{(11)}=0000010111 \underline{11}, \quad m_{1}\left(X^{(11)}\right)=56 \text {, } \\
& X^{(12)}=000001011 \underline{111}, \quad m_{1}\left(X^{(12)}\right)=56, \\
& X^{(13)}=00000101 \underline{11} \underline{1}, \quad m_{1}\left(X^{(13)}\right)=56 \text {, } \\
& X^{(14)}=0000010 \underline{11} 11 \underline{1}, \quad m_{1}\left(X^{(14)}\right)=56, \\
& X^{\left(d_{1}\right)}=X^{(\mathbf{1 5})}=000001 \underline{10111} \underline{1}, \quad m_{1}\left(X^{(\mathbf{1 5})}\right)=55 \text {, } \\
& X^{(16)}=00000110111 \underline{1}, \quad m_{1}\left(X^{(16)}\right)=55, \\
& X^{(17)}=0000 \underline{10} 10111 \underline{1}, \quad m_{1}\left(X^{(17)}\right)=54, \\
& X^{(18)}=000 \underline{10010111} \underline{1}, \quad m_{1}\left(X^{(18)}\right)=53 \text {, }
\end{aligned}
$$




$$
\begin{aligned}
& X^{(19)}=00 \underline{100010111} \underline{1}, \quad m_{1}\left(X^{(19)}\right)=52, \\
& X^{(20)}=0 \underline{1000010111} \underline{1}, \quad m_{1}\left(X^{(20)}\right)=51, \\
& X^{(21)}=\underline{10} 000010111 \underline{1}, \quad m_{1}\left(X^{(21)}\right)=50, \\
& X^{(22)}=\underline{10000010111 \underline{1},} m_{1}\left(X^{(22)}\right)=50, \\
& X^{(23)}=\underline{10000010111 \underline{1},} \quad m_{1}\left(X^{(23)}\right)=50, \\
& X^{(24)}=\underline{10000010111} \underline{1}, \quad m_{1}\left(X^{(24)}\right)=50, \\
& X^{(25)}=\underline{10000010111} \underline{1}, \quad m_{1}\left(X^{(25)}\right)=50, \\
& X^{(26)}=\underline{10000100111} \underline{1}, \quad m_{1}\left(X^{(26)}\right)=49, \\
& X^{\left(d_{2}\right)}=X^{(27)}=\underline{100001} \underline{00111} \underline{1}, \quad m_{1}\left(X^{(27)}\right)=49 \text {, } \\
& X^{(28)}=\underline{10000101011} \underline{1}, \quad m_{1}\left(X^{(28)}\right)=48, \\
& X^{(29)}=\underline{10000101} \underline{10} 1 \underline{1}, \quad m_{1}\left(X^{(29)}\right)=47, \\
& X^{(30)}=\underline{100001011} \underline{101}, \quad m_{1}\left(X^{(30)}\right)=46, \\
& X^{(31)}=\underline{100001011101}, \quad m_{1}\left(X^{(31)}\right)=46, \\
& X^{(32)}=\underline{100001011101}, \quad m_{1}\left(X^{(32)}\right)=46, \\
& X^{(33)}=\underline{100001 \underline{10}} 1 \underline{01}, \quad m_{1}\left(X^{(33)}\right)=45 \text {, } \\
& X^{(34)}=\underline{1000011011} \underline{01}, \quad m_{1}\left(X^{(34)}\right)=45, \\
& X^{(35)}=\underline{1000101011} \underline{01}, \quad m_{1}\left(X^{(35)}\right)=44,
\end{aligned}
$$

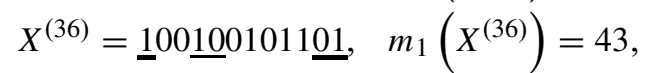

$$
\begin{aligned}
& X^{(38)}=\underline{1100001011 \underline{01},} m_{1}\left(X^{(38)}\right)=41, \\
& X^{\left(d_{3}\right)}=X^{(37)}=\underline{1010001011} \underline{01}, \quad m_{1}\left(X^{(37)}\right)=42 \text {, } \\
& X^{(39)}=\underline{110000101101}, \quad m_{1}\left(X^{(39)}\right)=41, \\
& X^{(40)}=\underline{110000101101}, \quad m_{1}\left(X^{(40)}\right)=41 \text {, } \\
& X^{(41)}=\underline{1100001011} \underline{01}, \quad m_{1}\left(X^{(41)}\right)=41, \\
& X^{(42)}=\underline{1100010011} \underline{01}, \quad m_{1}\left(X^{(42)}\right)=40, \\
& X^{(43)}=\underline{110001 \underline{001}} 1 \underline{01}, \quad m_{1}\left(X^{(43)}\right)=40, \\
& X^{(44)}=\underline{110001010101}, \quad m_{1}\left(X^{(44)}\right)=39, \longleftarrow \\
& X^{(45)}=\underline{11000101 \underline{1001},} \quad m_{1}\left(X^{(45)}\right)=38, \\
& X^{\left(d_{4}\right)}=X^{(\mathbf{4 6})}=\underline{1100010 \underline{1001}}, \quad m_{1}\left(X^{(\mathbf{4 6 )})}\right)=38 \text {, } \\
& X^{(47)}=\underline{110001 \underline{10}} \underline{001}, \quad m_{1}\left(X^{(47)}\right)=37 \text {, } \\
& X^{(48)}=\underline{110001101001}, \quad m_{1}\left(X^{(48)}\right)=37 \text {, } \\
& X^{(49)}=\underline{110010101001}, \quad m_{1}\left(X^{(49)}\right)=36, \\
& X^{(50)}=\underline{110} \underline{100101001}, \quad m_{1}\left(X^{(50)}\right)=35 \text {, } \\
& X^{(51)}=\underline{111000101001}, \quad m_{1}\left(X^{(51)}\right)=34, \\
& X^{(52)}=\underline{111000101001}, \quad m_{1}\left(X^{(52)}\right)=34 \text {, }
\end{aligned}
$$

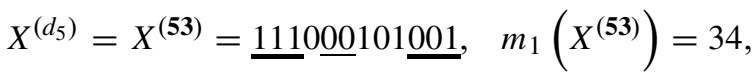

$$
\begin{aligned}
& X^{(54)}=\underline{111001001001}, \quad m_{1}\left(X^{(54)}\right)=33 \text {, }
\end{aligned}
$$

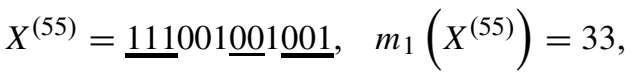

$$
\begin{aligned}
& X^{(56)}=\underline{1110010 \underline{10001}}, \quad m_{1}\left(X^{(56)}\right)=32 \text {, } \\
& X^{(57)}=\underline{111001} \underline{100001}, \quad m_{1}\left(X^{(57)}\right)=31 \text {, } \\
& X^{\left(d_{6}\right)}=X^{(\mathbf{5 8})}=\underline{11100 \underline{11}} \underline{00001}, \quad m_{1}\left(X^{(\mathbf{5 8})}\right)=31 \text {, } \\
& X^{(59)}=\underline{111010100001}, \quad m_{1}\left(X^{(59)}\right)=30 \text {, } \\
& X^{(60)}=\underline{111100100001}, \quad m_{1}\left(X^{(60)}\right)=29 \text {, } \\
& X^{\left(d_{7}\right)}=X^{(\mathbf{6 1})}=\underline{111100100001}, \quad m_{1}\left(X^{(\mathbf{6 1 )}}\right)=29 \text {, } \\
& X^{(62)}=\underline{1111} \underline{1000001}, \quad m_{1}\left(X^{(62)}\right)=28 \text {, } \\
& X^{(63)}=\underline{111101000001}, \quad m_{1}\left(X^{(63)}\right)=28 \text {, } \\
& X^{(64)}=\underline{1111} \underline{0100001}, \quad m_{1}\left(X^{(64)}\right)=29 \text {, } \\
& X^{(65)}=\underline{111100} \underline{00001}, \quad m_{1}\left(X^{(65)}\right)=29 \text {, } \\
& X^{(66)}=\underline{111101000001}, \quad m_{1}\left(X^{(66)}\right)=28 .
\end{aligned}
$$

Note that, unlike the simple encoding scheme in [20] where all the $k(k-1) / 2=66$ possible functions $X \rightarrow X^{(i)}$, $i \in[0, k(k-1) / 2)$, are used as the $m_{1}$-balancing functions, here only $p=8$ of them are used. In fact, the family of the $m_{1}$-balancing functions $\mathcal{B}=\left\{\left\langle\Gamma_{h}\right\rangle: h \in[0,7]\right\}$ is defined as follows. From the definition of $\Gamma_{h}$ in Table I, along with (10) and (12), we have $\left\langle\Gamma_{h}\right\rangle(X)=X^{\left(d_{h}\right)}, h \in[0,7]$, with $d_{0}=0$, $d_{1}=15, d_{2}=27, d_{3}=37, d_{4}=46, d_{5}=53, d_{6}=58$ and $d_{7}=61$. That is,

$$
\begin{aligned}
& \left\langle\Gamma_{0}\right\rangle(X)=X^{(\mathbf{0})}=x_{1} x_{2} x_{3} x_{4} x_{5} x_{6} x_{7} x_{8} x_{9} x_{10} x_{11} x_{12} \stackrel{\text { def }}{=} X, \\
& \left\langle\Gamma_{1}\right\rangle(X)=X^{(\mathbf{1 5})}=x_{2} x_{3} x_{4} x_{5} x_{6} x_{7} \underline{x_{12} x_{8}} x_{9} x_{10} x_{11} \underline{x_{1}}, \\
& \left\langle\Gamma_{2}\right\rangle(X)=X^{(27)}=\underline{x}_{12} x_{3} x_{4} x_{5} x_{6} x_{7} \underline{x_{8} x_{2}} x_{9} x_{10} x_{11} \underline{x_{1}}, \\
& \left\langle\Gamma_{3}\right\rangle(X)=X^{(37)}=\underline{x_{12}} x_{3} \underline{x_{11} x_{4}} x_{5} x_{6} x_{7} x_{8} x_{9} x_{10} \underline{x_{2} x_{1}},
\end{aligned}
$$

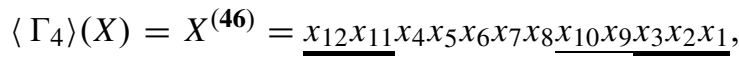

$$
\begin{aligned}
& \left\langle\Gamma_{5}\right\rangle(X)=X^{(53)}=\underline{x_{12} x_{11} x_{10}} x_{5} \underline{x_{6} x_{4}} x_{7} x_{8} x_{9} \underline{x_{3} x_{2} x_{1}}, \\
& \left\langle\Gamma_{6}\right\rangle(X)=X^{(\mathbf{5 8})}=\underline{x_{12} x_{11} x_{10}} x_{5} x_{6} \underline{x_{9} x_{7}} x_{8} \underline{x_{4} x_{3} x_{2} x_{1}},
\end{aligned}
$$

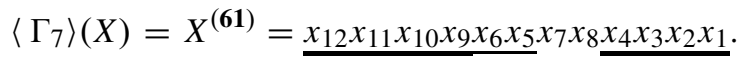

In this way, letting

$$
M_{1} \stackrel{\text { def }}{=} \frac{n(n+1)}{4}-\frac{k r}{2}=105-48=57,
$$

the encoding Algorithm 1 executes steps $S 1$ and $S 2$ as follows. $\mathbf{S 1}$ (for $h=0$ and $d_{0}=0$ ): compute

$$
\begin{aligned}
\left\langle\Gamma_{0}\right\rangle(X) & =X^{(0)}=100000101111(=X), \\
m_{1}\left(X^{(0)}\right) & =50, \\
\mu_{1} & =M_{1}-m_{1}\left(X^{(0)}\right)=57-50=7 .
\end{aligned}
$$

We have $\mu_{1}=7 \notin m_{1}\left(\Gamma_{0}\right)=[10,26] \Longleftrightarrow$ there is no check word $C \in \Gamma_{0}$ such that $m_{1}(C)=\mu_{1}$. 
TABLE I

EXAMPLE With $n=20, k=12, r=8$ AND $p=8$

\begin{tabular}{|c|c|c|c|c|c|c|c|c|c|}
\hline$S(8,4, \mu) \backslash \Gamma_{h}$ & $\Gamma_{0}$ & $\Gamma_{1}$ & $\Gamma_{2}$ & $\Gamma_{3}$ & $\Gamma_{4}$ & $\Gamma_{5}$ & $\Gamma_{6}$ & $\Gamma_{7}$ & $s(8,4, \mu)$ \\
\hline$S(8,4, \alpha=10)=$ & 11110000 & & & & & & & & 1 \\
\hline$S(8,4,11)=$ & 11101000 & & & & & & & & 1 \\
\hline$S(8,4,12)=$ & 11011000 & 11100100 & & & & & & & 2 \\
\hline$S(8,4,13)=$ & 10111000 & 11010100 & 11100010 & & & & & & 3 \\
\hline$S(8,4,14)=$ & $01111000 \quad 62$ & $10110100 \quad 11$ & 11001100 & 11010010 & 11100001,42 & & & & 5 \\
\hline$S(8,4,15)=$ & $0 1 1 1 0 1 0 0 \longdiv { 6 3 }$ & $10101100 \sqrt{12}$ & $10110010 \quad 24$ & $11001010 \quad 34$ & 11010001,43 & & & & 5 \\
\hline$S(8,4,16)=$ & $01101100 \quad 64$ & $10011100 \underline{13}$ & $01110010 \quad 25$ & $10101010 \quad 35$ & 11000110 & 10110001 51. & 110010015 & & 7 \\
\hline$S(8,4,17)=$ & $01011100 \quad 65$ & 01101010 14 & $10011010 \quad 26$ & 10100110 36 & 01110001,45 & 10101001 & 11000101 & & 7 \\
\hline$S(8,4,18)=$ & $00111100 \quad 0$ & $01011010 \lcm{15}$ & 01100110 & 10010110 37 & 01101001,46 & 10011001 & 10100101 & 1100001161 & 8 \\
\hline$S(8,4,19)=$ & $00111010 \quad 1$ & $0 1 0 1 0 1 1 0 \longdiv { 1 6 }$ & $1 0 0 0 1 1 1 0 \longdiv { 2 8 }$ & $0 1 0 1 1 0 0 1 \longdiv { 3 8 }$ & 01100101 47 & 10010101 54 & $10100011 \quad 59$ & & 7 \\
\hline$S(8,4,20)=$ & $00110110 \quad 2$ & $01001110 \quad \frac{17}{17}$ & $00111001 \quad 29$ & $\begin{array}{ll}01010101 & 39 \\
\end{array}$ & 10001101,48 & 01100011 & $10010011 \quad 60$ & & 7 \\
\hline$S(8,4,21)=$ & $00101110 \quad 3$ & $0 0 1 1 0 1 0 1 \longdiv { 1 8 }$ & 01001101 30 & 01010011 40 & $10001011 \lcm{49}$ & & & & 5 \\
\hline$S(8,4,22)=$ & $00011110 \quad 4$ & $00101101[19$ & 00110011 331 & 01001011 41 & 1000011150 & & & & 5 \\
\hline$S(8,4,23)=$ & $00011101 \quad 5$ & $00101011 \quad 20$ & 01000111 32 & & & & & & 3 \\
\hline$S(8,4,24)=$ & $00011011 \quad 6$ & $00100111_{21}$ & & & & & & & 2 \\
\hline$S(8,4,25)=$ & $00010111 \frac{7}{7}$ & & & & & & & & 1 \\
\hline$S(8,4, \beta=26)=$ & \begin{tabular}{|l|l|}
00001111 & \\
\end{tabular} & & & & & & & & 1 \\
\hline$\left|\Gamma_{h}\right|=$ & 17 & 13 & 11 & 9 & 9 & 5 & 5 & 1 & 70 \\
\hline$d_{h}=$ & 0 & 15 & 27 & 37 & 46 & 53 & 58 & 61 & $p=8$ \\
\hline
\end{tabular}

For any integer $\mu \in[10,26]$, the $(\mu-8)$ th row gives the vectors $C=c_{1} c_{2} \ldots c_{8} \in S(8,4, \mu)$ in ascending lexicographic order where $c_{1}$ is the least significant symbol, $c_{8}$ is the most significant symbol and $0<1$. The last column give $s(8,4, \mu)=|S(8,4, \mu)|$ in $(9)$, for all integers $\mu \in[10,26]$. For any integer $h \in[0,7]$, the $(h+2)$ th column gives $\Gamma_{h}$. The 19th and 20th rows give $\left|\Gamma_{h}\right|$ and $d_{h}$, for all $h \in[0,7]$, respectively. For all integers $\mu \in[10,26]$ and $h \in[0,7]$, if the entry in the lower right corner of the cell in the $(\mu-8)$ th row and $(h+2)$ th column is the integer $i \in[0,65]$ then $\hat{h}(i)=h$, where $\hat{h}:[0,65] \cap \mathbf{I N} \rightarrow[0,7]$ is the function in $(25)$.

S1 (for $h=1$ and $d_{1}=15$ ): compute

$$
\begin{aligned}
\left\langle\Gamma_{1}\right\rangle(X) & =X^{(15)}=000001 \underline{10111}, \\
m_{1}\left(X^{(15)}\right) & =55, \\
\mu_{1} & =M_{1}-m_{1}\left(X^{(15)}\right)=57-55=2 .
\end{aligned}
$$

We have $\mu_{1}=2 \notin m_{1}\left(\Gamma_{1}\right)=[12,24] \Longleftrightarrow$ there is no check word $C \in \Gamma_{1}$ such that $m_{1}(C)=\mu_{1}$.

S1 (for $h=2$ and $d_{2}=27$ ): compute

$$
\begin{aligned}
\left\langle\Gamma_{2}\right\rangle(X) & =X^{(27)}=\underline{10000100111}, \\
m_{1}\left(X^{(27)}\right) & =49, \\
\mu_{1} & =M_{1}-m_{1}\left(X^{(27)}\right)=57-49=8 .
\end{aligned}
$$

We have $\mu_{1}=8 \notin m_{1}\left(\Gamma_{2}\right)=[13,23] \Longleftrightarrow$ there is no check word $C \in \Gamma_{2}$ such that $m_{1}(C)=\mu_{1}$.

S1 (for $h=3$ and $d_{3}=37$ ): compute

$$
\begin{aligned}
\left\langle\Gamma_{3}\right\rangle(X) & =X^{(37)}=\underline{101000101101}, \\
m_{1}\left(X^{(37)}\right) & =42, \\
\mu_{1} & =M_{1}-m_{1}\left(X^{(37)}\right)=57-42=15 .
\end{aligned}
$$

The check word $C=11001010 \in \Gamma_{3}$ is such that $m_{1}(C)=$ $\mu_{1}=15 \in m_{1}\left(\Gamma_{3}\right)=[14,22]$. So, $h_{\text {bal }} \stackrel{\text { def }}{=} 3$ is an $m_{1}$-balancing index and the relative $m_{1}$-balancing check is $C_{3} \stackrel{\text { def }}{=} 11001010 \in \Gamma_{3}$.

S1 (for $h=4$ and $\left.d_{4}=46\right)$ : compute

$$
\begin{aligned}
\left\langle\Gamma_{4}\right\rangle(X) & =X^{(46)}=\underline{110001011001}, \\
m_{1}\left(X^{(46)}\right) & =38, \\
\mu_{1} & =M_{1}-m_{1}\left(X^{(46)}\right)=57-38=19 .
\end{aligned}
$$

The check word $C=01100101 \in \Gamma_{4}$ is such that $m_{1}(C)=\mu_{1}=19 \in m_{1}\left(\Gamma_{4}\right)=[14,22]$. So, $h_{b a l} \stackrel{\text { def }}{=} 4$ is an $m_{1}$-balancing index and the relative $m_{1}$-balancing check is $C_{4} \stackrel{\text { def }}{=} 01100101 \in \Gamma_{4}$.

S1 (for $h=5$ and $d_{5}=53$ ): compute

$$
\begin{aligned}
\operatorname{fun} \Gamma_{5}(X) & =X^{(53)}=\underline{111000101001}, \\
m_{1}\left(X^{(53)}\right) & =34, \\
\mu_{1} & =M_{1}-m_{1}\left(X^{(53)}\right)=57-34=23 .
\end{aligned}
$$

We have $\mu_{1}=23 \notin m_{1}\left(\Gamma_{5}\right)=[16,20] \Longleftrightarrow$ there is no check word $C \in \Gamma_{5}$ such that $m_{1}(C)=\mu_{1}$. 
TABLE II

Comparisons With the Optimal Codes AND the Codes In [20] FOR SOME CODE LENGTH VALUES $n \in 4 \mathbf{I N}$

\begin{tabular}{|c||c||c|c|c||c|c|c|}
\hline \multirow{2}{*}{$n$} & \multicolumn{1}{|c||}{ optimal } & \multicolumn{3}{c||}{ new scheme } & \multicolumn{3}{|c|}{ scheme in [20] } \\
\cline { 2 - 8 } & $\tilde{k}_{\text {op }}$ & $k$ & $\tilde{k}$ & $\Delta \tilde{k}_{\text {op }}$ & $k_{[20]}$ & $\tilde{k}_{[20]}$ & $\Delta \tilde{k}_{[20]}$ \\
\hline 4 & 1 & 2 & 1 & 0 & - & 1 & 0 \\
\hline 8 & 3 & 4 & 2 & 1 & - & 3 & -1 \\
\hline 12 & 5 & 6 & 4 & 1 & - & 5 & -1 \\
\hline 16 & 9 & 8 & 6 & 3 & - & 9 & -3 \\
\hline 20 & 12 & 12 & 9 & 3 & - & 12 & -3 \\
\hline 24 & 15 & 14 & 11 & 4 & - & 15 & -4 \\
\hline 28 & 19 & 18 & 15 & 4 & 12 & 9 & 6 \\
\hline 32 & 23 & 22 & 19 & 4 & 16 & 13 & 6 \\
\hline 36 & 26 & 24 & 21 & 5 & 20 & 17 & 4 \\
\hline 40 & 30 & 28 & 25 & 5 & 24 & 21 & 4 \\
\hline 44 & 34 & 32 & 29 & 5 & 28 & 25 & 4 \\
\hline 48 & 37 & 36 & 33 & 4 & 32 & 29 & 4 \\
\hline 52 & 41 & 40 & 37 & 4 & 32 & 29 & 8 \\
\hline 56 & 45 & 42 & 38 & 7 & 36 & 33 & 5 \\
\hline 60 & 49 & 46 & 42 & 7 & 40 & 37 & 5 \\
\hline 64 & 53 & 50 & 46 & 7 & 44 & 40 & 6 \\
\hline 128 & 115 & 112 & 108 & 7 & 104 & 100 & 8 \\
\hline 256 & 241 & 238 & 233 & 8 & 232 & 227 & 6 \\
\hline 512 & 495 & 492 & 487 & 8 & 476 & 471 & 16 \\
\hline 1024 & 1005 & 1002 & 996 & 9 & 988 & 982 & 14 \\
\hline 2048 & 2027 & 2024 & 2018 & 9 & 2008 & 2002 & 16 \\
\hline 4096 & 4073 & 4070 & 4063 & 10 & 4056 & 4049 & 14 \\
\hline 8192 & 8167 & 8164 & 8157 & 10 & 8148 & 8141 & 16 \\
\hline 16384 & 16357 & 16354 & 16346 & 11 & 16340 & 16332 & 14 \\
\hline 32768 & 32739 & 32736 & 32728 & 11 & 32720 & 32712 & 16 \\
\hline 65536 & 65505 & 65502 & 65493 & 12 & 65488 & 65479 & 14 \\
\hline
\end{tabular}

The first column is the overall length, $n \in 4 \mathbf{I N}$, of 2-OSN codes. The second column gives the information bits for the optimal 2-OSN code of length $n$. The third, fourth and fifth refer to the new coding scheme given in this paper. The sixth, seventh and eight refer to the coding scheme given in [20] In particular, for each coding scheme, $k$ is the maximum number of $m_{0^{-}}$ balanced bits that can be $m_{1}$-balanced into $n$ bits and $\tilde{k}$ is the maximum number of information bits that can be $m_{0}$-balanced into $k$ bits. In the fifth column, $\Delta \tilde{k}_{o p}=\tilde{k}_{o p}-\tilde{k}$. In the eigth column, $\Delta \tilde{k}_{[20]}=\tilde{k}-\tilde{k}_{[20]}$. For $n \geq 128$, the values of the second column are obtained with the approximation $|\mathcal{S} \mathcal{N}(n, 2)| \approx\left[(4 \sqrt{3} / \pi) 2^{n} / n^{2}\right]$ given in [20], [22].

S1 (for $h=6$ and $\left.d_{5}=58\right):$ compute

$$
\begin{aligned}
\left\langle\Gamma_{6}\right\rangle(X) & =X^{(58)}=\underline{111001100001}, \\
m_{1}\left(X^{(58)}\right) & =31, \\
\mu_{1} & =M_{1}-m_{1}\left(X^{(58)}\right)=57-31=26 .
\end{aligned}
$$

We have $\mu_{1}=26 \notin m_{1}\left(\Gamma_{6}\right)=[16,20] \Longleftrightarrow$ there is no check word $C \in \Gamma_{6}$ such that $m_{1}(C)=\mu_{1}$.

S1 (for $h=7$ and $\left.d_{5}=61\right)$ : compute

$$
\begin{aligned}
\left\langle\Gamma_{7}\right\rangle(X) & =X^{(61)}=\underline{111100100001}, \\
m_{1}\left(X^{(61)}\right) & =29, \\
\mu_{1} & =M_{1}-m_{1}\left(X^{(58)}\right)=57-29=28 .
\end{aligned}
$$

We have $\mu_{1}=28 \notin m_{1}\left(\Gamma_{7}\right)=[18,18] \Longleftrightarrow$ there is no check word $C \in \Gamma_{7}$ such that $m_{1}(C)=\mu_{1}$.

At this point, execute step $S 2$.

S2: For example, select as encoding of the $m_{0}$-balanced word $X$ the $m_{1}$-balanced word

$$
\mathcal{E}_{2}(X)=X^{(37)} C_{3}=\underline{1010001011} \underline{01} 11001010 .
$$

However, note from the above, that $\mathcal{E}_{2}(X)=X^{(46)} C_{4}$ can also be chosen because

$$
h_{b a l}(X)=\{3,4\} \subseteq[0,7] .
$$

With regard to decoding, on receiving the 2-OSN word

$$
Y C=10100010110111001010,
$$

the sequence of the last $r=8$ bits (i.e., $C=11001010$ ) is the check word that allows to identify the $m_{1}$-balancing function $\left\langle\Gamma_{h}\right\rangle$ used in the encoding process. Since $C \in \Gamma_{3}$, the remaining word $Y$ is decoded into the $m_{0}$-balanced word

$$
\mathcal{E}_{2}^{-1}(Y C)=\left\langle\Gamma_{3}\right\rangle^{-1}(Y)=100000101111
$$

\section{EFFiCIENT IMPLEMENTATIONS OF THE REFINED CODING SCHEME AND THE COMPLEXITY ANALYSIS}

There may be many ways to implement the coding scheme defined in Section II. However, before going into the details, note that given any integer $d \in[0, k(k-1) / 2]$ and word $X=x_{1} x_{2} \ldots x_{n} \in \mathbf{Z}_{2}^{k}$ the word $X^{(d)} \in \mathbf{Z}_{2}^{k}$ can be computed in $O(k \log k)$ bit operations with $O(k)$ bit memory elements with a sequence of at most $k / 2$ "giant steps" followed by a sequence of at most $2 k-3$ "baby steps"; as done in [20]. In the sequence of "giant steps", for $j=1,2, \ldots$, the bit $x_{j}$ is exchanged with the bit $x_{k-j+1}$ to compute $X^{\left(i_{j}\right)}$ until $i_{j+1}>d$, where

$$
i_{j+1}=i_{j}+2 k-4 j-3
$$

and $i_{0}=0$. As soon as $i_{j+1}>d$, the sequence of "baby steps" follows to compute $X^{\left(i_{j}+1\right)}, X^{\left(i_{j}+2\right)}, \ldots, X^{(d)}$ from $X^{\left(i_{j}\right)}$. Analogously, given $X^{(d)} \in \mathbf{Z}_{2}^{k}$ and $d \in[0, k(k-1) / 2]$ the word $X \in \mathbf{Z}_{2}^{k}$ can be recovered in $O(k \log k)$ bit operations with $O(k)$ bit memory elements. In this section, we rely on these giant-baby step based algorithms.

Now, a simple way to implement the coding scheme in Section II, relies on computing the entire random walk sequence

$R W S(X) \stackrel{\text { def }}{=}\left\{\left(i, X^{(i)}, m_{1}\left(X^{(i)}\right)\right): i=0,1, \ldots, \frac{k(k-1)}{2}\right\}$

during the encoding. In this process, whenever $i=d_{h}$, for $h=0,1, \ldots, p-1$, step S1 of Algorithm 1 is executed to check whether $\mu_{1} \in m_{1}\left(\Gamma_{h}\right)=\left[\alpha_{h}, \beta_{h}\right]$; that is, if there exists an $m_{1}$-balancing check $C \in \Gamma_{h}$. If $\mu_{1} \in\left[\alpha_{h}, \beta_{h}\right]$ then $h_{b a l}=h, d_{h_{b a l}}=i$ and the information part $Y=$ $\left\langle\Gamma_{h_{b a l}}\right\rangle(X)=X^{(i)}$ is readily available to form a codeword. With regard to the check word, $C_{h_{b a l}} \in \Gamma_{h_{b a l}}$, to be appended to $Y$, it can be computed with a table look-up, like the example Table I, indexed by the readily available index $h$ and the readily available $m_{1}$-unbalance $\mu_{1}$ in (24). It is readily seen 
that such strategy essentially takes time $T=O\left(k^{2} \log k\right)$ bit operations to compute the entire random walk sequence $R W S(X)$. With regard to the space complexity, if we assume that the partition $\mathcal{C S}$ in Definition 1 is chosen so that (18) holds with equality then

$$
S=O\left(\left|\bigcup_{h=0}^{p-1} \Gamma_{h}\right| \cdot r\right)=O\left(k^{2} r\right)
$$

memory bits are essentially needed to store the check word table. With regard to the decoding, for simplicity, assume that the function $\left(i, X^{(i)}\right) \rightarrow X$ is computed sequentially with $T=$ $O(k \log k)$ bit operations and $S=O(k)$ memory bits as the giant-baby step based decoding algorithm in [20]. In this case, on receiving a codeword $Y C=X^{\left(d_{h_{b a l}}\right)} C$, a table look-up indexed by $C$ can be maintained to compute $i=d_{h_{b a l}}$ from $C$. Once $d_{h_{b a l}}$ is known, $X$ can be computed from $\left(d_{h_{b a l}}, Y\right)$ with the giant-baby step based algorithm explained above. In this way, $T=O(k \log k)$ bit operations are essentially needed to compute $X$ from $\left(d_{h_{\text {bal }}}, Y\right)$ and $S=O\left(k^{2} \log k\right)$ memory bits are essentially needed to compute $d_{h_{b a l}} \in[0, k(k-1) / 2]$ from $C \in \bigcup_{h=0}^{p-1} \Gamma_{h}$ with the table look-up. Note that, if the codes are close to optimal then $r=O(\log k)$ because of (3). In this case, this implementation requires $T=O\left(k^{2} \log k\right)$ bit operations with $S=O\left(k^{2} \log k\right)$ memory bits for encoding and $T=O(k \log k)$ bit operations with $S=O\left(k^{2} \log k\right)$ memory bits for decoding. Also, note that with the above complexities all possible encodings of a given $m_{0}$-balanced data word can be computed and, as mentioned earlier, this may be useful to convey some more extra information as done in [12], [16], and [29] for balanced codes.

When $k$ is relatively very big, the above implementation may result prohibitively very complex in terms of time and space. In the remaing part of this section, we show another implementation of the coding scheme presented in Section II which computes one codeword for any given $m_{0}$-balanced data word and requires $O(k \log k)$ bit operations with $O(k)$ bit memory elements, as claimed in the abstract. Two major coding problems need to be addressed and efficiently solved in the General Encoding Algorithm 1 for a given $m_{0}$-balanced data word $X$ :

1) find one balancing index $h_{b a l} \in h_{\text {bal }}(X)$ and compute the relative information part $Y=\left\langle\Gamma_{h_{b a l}}\right\rangle(X)=X^{\left(d_{h_{b a l}}\right)}$; and

2) compute the relative check word $C_{h_{b a l}} \in \Gamma_{h_{b a l}}$ to be appended to $Y$.

Here, instead of going into details in designing efficient algorithms directly solving the first problem, we efficiently reduce this problem to the coding problem of finding an $m_{1}$-crossing index $i_{c x}$ of $X$ defined in Definition 2. In this reduction, from a given $i_{c x}$, the balancing index $h_{b a l}$ is computed easily with Algorithm 3 below as the balancing index, $\hat{h} \in[0, p-1]$, defined in the proof of Theorem 2. Algorithm 3 also efficiently computes $d=d_{h_{b a l}}$. The information part $Y$ is then computed from $d$ and $X$ with the above giant-baby step method. With regard to the second problem, by using the enumerative coding technique in [4], the check word $C_{h_{b a l}}$ of the codeword is computed as the $\left(h_{b a l}+1\right)$ th element in $S\left(r, r / 2, \mu_{1}\right)$, with $\mu_{1}$ being the $m_{1}$-unbalance in (24). All this can be done by maintaining a table look-up of size $O\left((\log k)^{5}\right)$ bit memory elements. In Sub-section III-A we give the encoding algorithm with the complexity analysis and in Sub-section III-B we analyze the associated decoding algorithm. In the following, for simplicity, assume $S(r, r / 2)=\bigcup_{h=0}^{p-1}\left|\Gamma_{h}\right|$.

\section{A. Encoding}

Refer to Table I for an example. Let

$$
\hat{h}:\left[0, \frac{k(k-1)}{2}+\epsilon(k, r)\right) \cap \mathbf{N} \rightarrow[0, p-1]
$$

be the integer value function defined as

$$
\hat{h}(i) \stackrel{\text { def }}{=}\left\{\begin{array}{l}
\hat{h} \text { if } i \in I_{\hat{h}} \text { and } \hat{h} \in[1, p-1] \\
0 \text { if } i \in I_{0} \cup I_{p}
\end{array}\right.
$$

where $\epsilon(k, r)$ is defined in (18) and the integer intervals $I_{0}$, $I_{1}, \ldots, I_{p}$ are defined in (11). Note that, if (18) holds then $\hat{h}(i)$ is a well defined integer function such that

1) there exists an $m_{1}$-crossing index, $i_{c x} \in[0, k(k-1) / 2+\epsilon(k, r))$ according to Definition 2;

and

2) if $i_{c x} \in[0, k(k-1) / 2+\epsilon(k, r))$ is an $m_{1}$-crossing index then $\hat{h}\left(i_{c x}\right) \in[0, p-1]$ is a balancing index with respect to the refined scheme. In fact, $\hat{h}\left(i_{c x}\right)$ is exactly equal to the integer, $\hat{h}$, defined in the proof of Theorem 2.

For example, in Table I, $\hat{h}(0-8)=0, \hat{h}(9-21)=1, \ldots$, $\hat{h}(61)=7$ and $\hat{h}(62-65)=0$. Also, for the $m_{0}$-balanced information word $X$ in Example $5, i_{c x}=44$ is an $m_{1}$ crossing index and so $\hat{h}(44)=4$ is a refined scheme balancing index. Now, given any $i \in[0, k(k-1) / 2+\epsilon(k, r))$, the indices $\hat{h}(i)$ and $d_{\hat{h}(i)}$ can be computed efficiently with Algorithm 3 below, whose idea is to compute partial areas of the "bell-shaped histogram" in Table I. In the algorithm, refer to Theorem 3 in the Appendix, Table I and note that

$$
\begin{aligned}
& \alpha=\min _{C \in S(r, r / 2)} m_{1}(C)=\frac{r(r+2)}{8} \in \mathbf{I N}, \\
& \beta=\max _{C \in S(r, r / 2)} m_{1}(C)=\alpha+\frac{r^{2}}{4} \in \mathbf{I N} .
\end{aligned}
$$

Also, for simplicity, assume to have a table look-up of size $O\left(r^{3}\right)$ bits which stores the integer sequence

$$
\left\{s(\mu) \stackrel{\text { def }}{=} s(r, r / 2, \mu): \mu \in\left[\alpha-1,\left\lfloor\frac{\alpha+\beta}{2}\right\rfloor\right] \cap \mathbf{I N}\right\}
$$

of the $O\left(r^{2}\right)$ integer numbers defined in (9), each of which of size $r$ bits. Furthermore, let

$$
i_{0}(\alpha-1)=-\left\lceil\frac{\left|\Gamma_{0}\right|}{2}\right\rceil
$$


and, for all integer $\mu \in[\alpha,\lfloor(\alpha+\beta) / 2\rfloor]$, let

$$
\begin{aligned}
i_{0}(\mu) & \stackrel{\text { def }}{=} \sum_{h=0}^{s(\mu)-1}\left|\Gamma_{h}\right|-\left\lceil\frac{\left|\Gamma_{0}\right|}{2}\right\rceil \\
& =\left(\sum_{h=0}^{s(\mu-1)-1}\left|\Gamma_{h}\right|-\left\lceil\frac{\left|\Gamma_{0}\right|}{2}\right\rceil\right)+\sum_{h=s(\mu-1)}^{s(\mu)-1}\left|\Gamma_{h}\right| \\
& =i_{0}(\mu-1)+\sum_{h=s(\mu-1)}^{s(\mu)-1}\left|\Gamma_{h}\right| .
\end{aligned}
$$

Note that, 1) the quantity " $i_{0}(\mu)+\left\lceil\left|\Gamma_{0}\right| / 2\right\rceil=\sum_{h=0}^{s(\mu)-1}\left|\Gamma_{h}\right|$ is equal to the area of the bell-shaped histogram in Table I until the $(\mu-\alpha+1)$ th lower corner (in the order from left to right)"; 2) the integer interval

$$
\begin{aligned}
I_{s(\mu)} & =\left[d_{s(\mu)}-\left\lceil\frac{\left|\Gamma_{s(\mu)}\right|}{2}\right\rceil+1, d_{s(\mu)}+\left\lfloor\frac{\left|\Gamma_{s(\mu)}\right|}{2}\right]\right] \\
& =\left[i_{0}(\mu)+1, i_{0}(\mu)+\left|\Gamma_{s(\mu)}\right|\right]
\end{aligned}
$$

because of (10) and (11); and, 3) if $h \in[s(\mu-1), s(\mu))$ then the quantity $\left|\Gamma_{h}\right|$ is constant and

$$
\left|\Gamma_{h}\right|=\left|\Gamma_{0}\right|-2(\mu-\alpha)=(\beta-\alpha+1)-2(\mu-\alpha) .
$$

So, (29), (30) and (32) give,

$$
i_{0}(\mu)=\left\{\begin{array}{lc}
-\left\lceil\left|\Gamma_{0}\right| / 2\right\rceil & \text { if } \mu=\alpha-1, \\
i_{0}(\mu-1)+[s(\mu)-s(\mu-1)]\left[\left|\Gamma_{0}\right|-2(\mu-\alpha)\right] & \text { if } \mu \in[\alpha,\lfloor(\alpha+\beta) / 2\rfloor] .
\end{array}\right.
$$

Algorithm 3 to compute $\hat{h}(i)$ and relative $d_{\hat{h}}$ from $i$

Input: the integer $i \in[0, k(k-1) / 2+\epsilon(k, r))$.

Output: the integer $\hat{h}(i) \in[0, p-1]$ defined in (25) and $d_{\hat{h}} \in\left\{d_{h}: h=0,1, \ldots, p-1\right\}$, where the integers $d_{h}$ are defined in (10).

Perform steps S0, S1, S2 and S3.

S0: if

$$
\begin{aligned}
i \in\left[0,\left\lfloor\frac{\left|\Gamma_{0}\right|}{2}\right\rfloor\right] \\
\cup\left[\left\lfloor\frac{\left|\Gamma_{0}\right|}{2}\right\rfloor+\sum_{h=1}^{p-1}\left|\Gamma_{h}\right|+1, \frac{k(k-1)}{2}+\epsilon(k, r)\right)
\end{aligned}
$$

then let $\hat{h}=0$ and $d_{\hat{h}}=0$ according to the definition (25) of $\hat{h}$. Hence, go to step S3.

S1: compute the largest integer $\hat{\mu} \in[\alpha,\lfloor(\alpha+\beta) / 2\rfloor]$ such that $i_{0}(\hat{\mu})<i$, where the function $i_{0}(\mu)$ is given in (33). Also, compute $i_{0}(\hat{\mu})$. Note that for such $\hat{\mu}$ we have $i_{0}(\hat{\mu})<$ $i \leq i_{0}(\hat{\mu}+1)$.

S2: according to (31) and (32), execute the steps S2.1, S2.2, S2.3 and S2.4.

S2.1: compute $\gamma=\left|\Gamma_{\hat{h}}\right|$ as follows.

$$
\gamma=\left|\Gamma_{0}\right|-2[(\hat{\mu}+1)-\alpha] .
$$

S2.2: compute the quotient

$$
\phi=\left\lfloor\frac{i-i_{0}(\hat{\mu})-1}{\gamma}\right\rfloor .
$$

S2.3: compute

$$
\hat{h}=s(\hat{\mu})+\phi .
$$

S2.4: compute

$$
d_{\hat{h}}=i_{0}(\hat{\mu})+\phi \cdot \gamma+\left\lceil\frac{\gamma}{2}\right\rceil
$$

S3: output $\hat{h}, d_{\hat{h}}$ and exit.

Example 6 [For $k=12, r=8$ and $p=8$ (Continued)]: In the example given in Table I, if $i=44 \in[0,65]$ is given as input to Algorithm 3 then it executes as follows.

S0: since $i=44 \notin[0,8] \cup[62,65]$ continue to step S1.

S1: compute the largest integer $\hat{\mu} \in[\alpha,\lfloor(\alpha+\beta) / 2\rfloor]=$ $[10,18]$ such that $i_{0}(\hat{\mu})<i$. From $(33)$, the encoder computes

$$
\begin{aligned}
i_{0}(\alpha) & =-9+(1-0)(17)=8<44, \\
i_{0}(\alpha+1) & =8+(1-1)(15)=8<44, \\
i_{0}(\alpha+2) & =8+(2-1)(13)=21<44, \\
i_{0}(\alpha+3) & =21+(3-2)(11)=32<44, \\
i_{0}(\alpha+4) & =32+(5-3)(9)=50 \geq 44 .
\end{aligned}
$$

So, $\hat{\mu}=\alpha+3=13$.

S2: execute the steps S2.1, S2.2, S2.3 and S2.4.

S2.1: compute $\gamma=17-2(14-10)=9$.

S2.2: compute

$$
\phi=\left\lfloor\frac{44-32-1}{9}\right\rfloor=\left\lfloor\frac{11}{9}\right\rfloor=1 .
$$

S2.3: compute $\hat{h}=s(13)+1=4$.

S2.4: compute $d_{\hat{h}}=32+1 \cdot 9+\lceil 9 / 2\rceil=46$.

S3: output $\hat{h}=4, d_{\hat{h}}=46$ and exit.

With regard to the space complexity of Algorithm 3, the predominant term is the size of the table look-up storing the integer sequence (28). Hence, Algorithm 3 requires a size of $O\left(r^{3}\right)$ bits. With regard to the time complexity, note that step S0 can be accomplished in time $O(r)$ bit operations because the size of $i=O\left(k^{2}\right)$ is $O(\log i)=$ $O(\log k)=O(r) ; \mathrm{S} 1$ can be accomplished in time $O\left(r^{3} \log r\right)$ bit operations because, with (33), S1 can be computed with $O(\beta-\alpha)=O\left(r^{2}\right)$ substeps (see (27)), each of which performing $O\left(\log \left|\Gamma_{0}\right|\right)=O(\log (\beta-\alpha))=O(\log r)$ additions (see (32)) of numbers of size $O(\log i)=O(r)$ bits; S2 can be accomplished in time $O\left(r^{2}\right)$ because step S2.1 takes time $O(\log r)$ bit operations, S2.2 takes time $O\left(\log ^{2} k\right)=O\left(r^{2}\right)$ bit operations, S2.3 takes time $O(r)$ bit operations, S2.4 takes time $O\left(\log ^{2} k\right)=O\left(r^{2}\right)$ bit operations; and, finally, step S3 takes time $O(1)$ bit operations. Hence, Algorithm 3 requires a time complexity of $O\left(r^{3} \log r\right)$ bit operations.

At this point, using Algorithm 3 and the enumerative coding technique in [4] the efficient encoding Algorithm 4 can be given below. So, as in Table I, let us assume that the check 
words in each $S(r, r / 2, \mu)$ are arranged in some lexicographic order. In Algorithm 4, once a balancing index $h_{b a l}$ is chosen, say, by using property (26), as $h_{b a l}=\hat{h}\left(i_{c x}\right)$, the check word is computed in step S1.5 as the $\left(h_{b a l}+1\right)$ th element in $S\left(r, r / 2, \mu_{1}\right)$, with $\mu_{1}$ being the quantity which makes the overall codeword $m_{1}$-balanced. To this aim, for simplicity, assume to have a table look-up of size $O\left(r^{5}\right)$ bits which stores the set

$$
\{s(a, b, c): a, b \in[0, r] \text { and } c \in[0, r(r+1) / 2]\}
$$

of $O\left(r^{4}\right)$ integer numbers in (9) each of which has size $r$ bits.

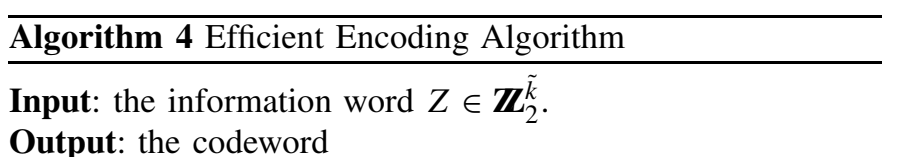

$$
Y C=\mathcal{E}_{2}\left(\mathcal{E}_{1}(Z)\right)=\left(\mathcal{E}_{1} \circ \mathcal{E}_{2}\right)(Z) \in \mathcal{S N}(n, 2)
$$

where $n=k+r, Y \in \mathbf{Z}_{2}^{k}$ and $C \in \mathbf{Z}_{2}^{r}$.

Perform steps S0, S1, S2 and S3.

SO apply any $m_{0}$-balancing method to $Z$ and let $X$ be the $m_{0}$-balanced word of length $k$ associated with $Z$, i.e. $X=\mathcal{E}_{1}(Z)$. In this way, $m_{0}(X)=k / 2$.

S1 (find the balancing index of $X$ and compute the check word): execute the steps S1.1-S1.5.

S1.1: find an $m_{1}$-crossing index of $X$ according to Definition 2.

S1.2: compute $h_{b a l}=\hat{h}\left(i_{c x}\right)$ and the relative $d_{h_{b a l}}$ with Algorithm 3.

S1.3: compute $\left\langle\Gamma_{h_{b a l}}\right\rangle(X)=X^{\left(d_{h_{b a l}}\right)}$ and $m_{1}\left(\left\langle\Gamma_{h_{b a l}}\right\rangle(X)\right)$.

S1.4: compute

$$
\mu_{1}=\frac{n(n+1)}{4}-m_{1}\left(\left\langle\Gamma_{h_{b a l}}\right\rangle(X)\right)-\frac{k r}{2} .
$$

S1.5: With the enumerative coding technique, compute the $\left(h_{b a l}+1\right)$ th element in $S\left(r, r / 2, \mu_{1}\right)$. Let it be $C_{h_{b a l}, \mu_{1}}$.

S2: Since $h_{b a l}$ is a balancing index of $X$, select the $m_{1}$ balanced encoding word of $X$ as

$$
\mathcal{E}_{2}(X)=Y C=\left\langle\Gamma_{h_{b a l}}\right\rangle(X) C_{h_{\text {bal }}, \mu_{1}}=X^{\left(d_{h_{b a l}}\right)} C_{h_{\text {bal }}, \mu_{1}}
$$

In this way the data word $Z$ is encoded as $\mathcal{E}_{2}(X)=Y C=$ $\mathcal{E}_{2}\left(\mathcal{E}_{1}(Z)\right)$.

S3: output $\mathcal{E}_{2}(X)=X^{\left(d_{h_{b a l}}\right)} C_{h_{b a l}, \mu_{1}}$ and exit.

Example 7 [For $k=12, r=8$ and $p=8$ (Continued)]: As in Example 5, suppose $X=100000101111$ is the given $m_{0}$-balanced word which needs to be encoded into a word of $\mathcal{S N}(20,2)$. In this case, Algorithm 4 executes as follows.

S0 by applying the choosen $m_{0}$-balancing method to $Z$, the output is $X=100000101111$.

S1 (find the balancing index of $X$ and compute the check word): execute the steps S1.1-S1.5.

S1.1: find an $m_{1}$-crossing index of $X$. From the random walk in Example 5, $i_{c x}=44 \in[0,65]$ is such that $m_{1}\left(X^{\left(i_{c x}\right)}\right)=$ $k(k+1) / 4=39$. Note that, for the chosen example word $X$ there is only one $m_{1}$-crossing index. But, in general, there may be many such indices.
S1.2: compute $h_{b a l}=\hat{h}\left(i_{c x}\right)$ and the relative $d_{h_{b a l}}$ with Algorithm 3. From Example 6,

$$
h_{b a l}=\hat{h}(44)=4
$$

and

$$
d_{h_{b a l}}=d_{4}=46 .
$$

S1.3: compute

$$
\left\langle\Gamma_{4}\right\rangle(X)=X^{(46)}=\underline{1100010 \underline{1001}}
$$

and

$$
m_{1}\left(\left\langle\Gamma_{4}\right\rangle(X)\right)=38 .
$$

S1.4: compute

$\mu_{1}=\frac{n(n+1)}{4}-m_{1}\left(\left\langle\Gamma_{4}\right\rangle(X)\right)-\frac{k r}{2}=105-38-48=19$.

S1.5: With the enumerative coding technique, compute the $\left(h_{\text {bal }}+1\right)$ th $=5$ th element in $S(8,4,19)$. It is (see Table I)

$$
C_{4,19}=01100101 \text {. }
$$

S2: Compute

$$
\begin{aligned}
& \mathcal{E}_{2}(X)=Y C=X^{(46)} C_{4,19} \\
& =\underline{110001011001} 01100101 \in \mathcal{S N}(20,2) \text {. }
\end{aligned}
$$

S3: Output $\mathcal{E}_{2}(X)=11000101100101100101$ and exit.

With regard to the complexity of Algorithm 4, note that step S0 can be accomplished in space $O(k)$ memory bits and time $O(k \log k)$ bit operations by using any of the methods given in [1], [2], [7], [11], [12], [16], [18], [19], [21], and [29]. $\mathrm{S} 1$ can be accomplished in space $O\left(r^{5}+k\right)$ memory bits and time $O\left(r^{3}\right)$ bit operations because step S1.1 takes $O(k)$ memory bits and $O(k \log k)$ bit operations if done with the giant-baby step method explained at the beginning of this section, S1.2 takes $O\left(r^{3}\right)$ memory bits and $O\left(r^{3} \log r\right)$ bit operations, S1.3 takes $O(k)$ memory bits and $O(k \log k)$ bit operations if done with the giant-baby step method, S1.4 takes $O(\log k)$ memory bits and $O(\log k)$ bit operations, S1.5 takes $O(r)$ additions of $r$-bit numbers so, with the table look-up (34) of size $O\left(r^{5}\right)$ memory bits, it takes time $O\left(r^{2}\right)$ bit operations; and, finally, step S2 takes time $O(1)$ bit operations. Hence, Algorithm 4 has a space complexity of $O\left(r^{5}+k\right)=O(k)$ memory bits and a time complexity of $O\left(r^{3} \log r+k \log k\right)=$ $O(k \log k)$ bit operations because of (3).

\section{B. Decoding}

In Algorithm 4 the check word $C$ encodes the balancing index $h_{b a l}$ through the index of $C$ in the lexicographically ordered set $S\left(r, r / 2, m_{1}(C)\right)$. Hence, during the decoding process, once this lexicogrphic index $h_{b a l}$ is computed, an efficient algorithm is needed which computes $d_{h_{b a l}}$ from $h_{b a l}$. Once $d_{h_{b a l}}$ is known, $X$ can be recovered easily from $d_{h_{b a l}}$ and $Y$ with the giant-baby step method of [20]. The algorithm which computes $d_{\hat{h}}$ from any $\hat{h}$ is given below. As in Algorithm 3, it is assumed to have a table look-up storing the integer sequence in (28). 


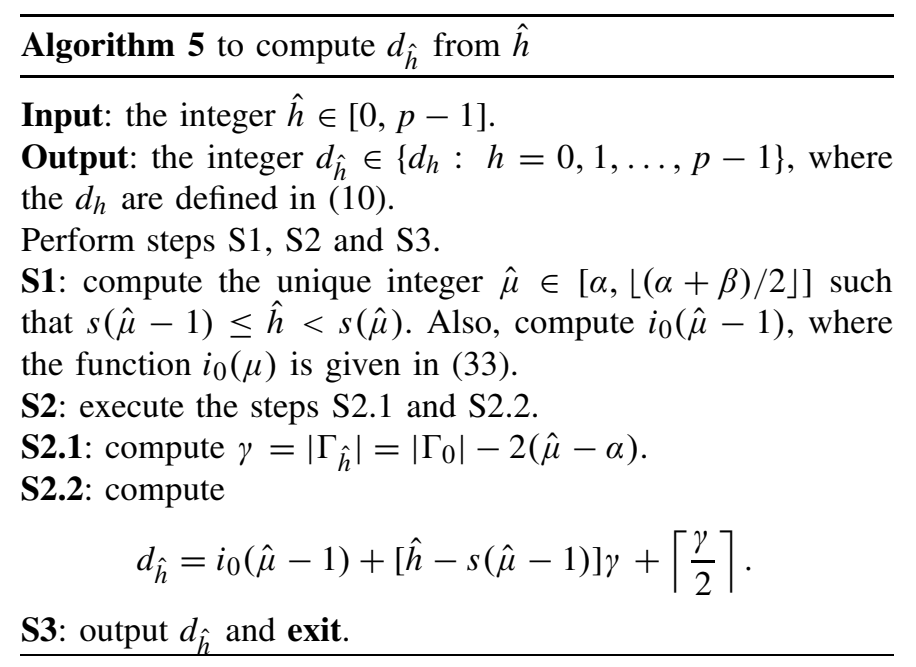

Example 8 [For $k=12, r=8$ and $p=8$ (Continued)]: In the example given in Table $I$, if $\hat{h}=4 \in[0,7]$ is given as input to Algorithm 5 then it executes as follows.

S1: compute the unique integer $\hat{\mu} \in[\alpha,\lfloor(\alpha+\beta) / 2\rfloor]=$ $[10,18]$ such that $s(\hat{\mu}-1) \leq \hat{h}<s(\hat{\mu})$. Also, compute $i_{0}(\hat{\mu}-1)$. From Table I and (33), the decoder computes

$$
\begin{gathered}
\hat{h}=4 \notin[s(9), s(10))=[0,1) \text { and } \\
i_{0}(\alpha-1)=-9, \\
\hat{h}=4 \notin[s(10), s(11))=[1,1) \text { and } \\
i_{0}(\alpha)=-9+(1-0)(17)=8, \\
\hat{h}=4 \notin[s(11), s(12))=[1,2) \text { and } \\
i_{0}(\alpha+1)=8+(1-1)(15)=8, \\
\hat{h}=4 \notin[s(12), s(13))=[2,3) \text { and } \\
i_{0}(\alpha+2)=8+(2-1)(13)=21, \\
\hat{h}=4 \in[s(13), s(14))=[3,5) \text { and } \\
i_{0}(\alpha+3)=21+(3-2)(11)=32 .
\end{gathered}
$$

So, $\hat{\mu}=\alpha+4=14$.

S2: execute the steps $S 2.1$ and $S 2.2$.

S2.1: compute

$$
\gamma=\left|\Gamma_{\hat{h}}\right|=\left|\Gamma_{0}\right|-2(\hat{\mu}-\alpha)=17-2(14-10)=9 .
$$

S2.2: compute

$$
\begin{aligned}
d_{\hat{h}} & =i_{0}(\hat{\mu}-1)+[\hat{h}-s(\hat{\mu}-1)] \gamma+\left\lceil\frac{\gamma}{2}\right\rceil \\
& =32+(4-3) \cdot 9+5=46 .
\end{aligned}
$$

S3: output $d_{\hat{h}}=46$ and exit.

As the above complexity analysis of Algorithm 3, we conclude that Algorithm 5 can execute with $O\left(r^{3}\right)$ memory bits and $O\left(r^{3} \log r\right)$ bit operations.

At this point, the decoding algorithm can be given below. As Algorithm 4, assume to have a table look-up of size $O\left(r^{5}\right)$ bits storing the set (34) of integers.

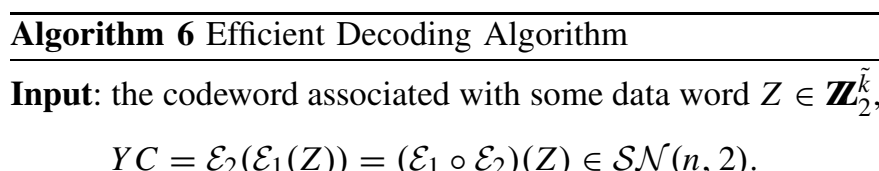

with $n=k+r, Y \in \mathbf{Z}_{2}^{k}$ and $C \in \mathbf{Z}_{2}^{r}$.

Output: the information word $Z=\left(\mathcal{E}_{1} \circ \mathcal{E}_{2}\right)^{-1}(Y C)$.

Perform steps S0, S1, S2 and S3.

S0: compute the index $h_{b a l} \in[0, p-1]$ such that $C \in \Gamma_{h_{b a l}}$. Perform steps S0.1, S0.2 and S0.3.

S0.1: compute $\mu_{1}=m_{1}(C)$.

S0.2: compute the lexicographic index, say

$$
\xi_{\mu_{1}}(C) \in\left[0, s\left(r, r / 2, \mu_{1}\right)\right),
$$

of $C$ in $S\left(r, r / 2, \mu_{1}\right)$.

S0.3: set

$$
h_{b a l}=\xi_{\mu_{1}}(C) \in\left[0, s\left(r, r / 2, \mu_{1}\right)\right) \subseteq[0, p-1] .
$$

S1: compute the word

$$
X=\mathcal{E}_{2}^{-1}(Y C)=\left\langle\Gamma_{h_{\text {bal }}}\right\rangle^{-1}(Y) .
$$

Perform steps S1.1, S1.2.

S1.1: set $\hat{h}=h_{\text {bal }} \in[0, p-1]$ and run Algorithm 5 to compute

$$
d_{\hat{h}}=d_{h_{b a l}} \in\left[0, \frac{k(k-1)}{2}+\epsilon(k, r)\right)
$$

from $\hat{h}$.

S1.2: from $d_{h_{b a l}}$, compute the word $X=\left\langle\Gamma_{h_{b a l}}\right\rangle^{-1}(Y)$ with the giant-baby step method in [20].

S2: undo the $m_{0}$-balancing encoding chosen in step S0 of Algorithm 4 on the word $X$. Let $Z=\mathcal{E}_{1}^{-1}(X) \in \mathbf{Z}_{2}^{\tilde{k}}$ be the information word whose $m_{0}$-balanced encoding is $X$. S3: output the word

$$
Z=\mathcal{E}_{1}^{-1}(X)=\mathcal{E}_{1}^{-1}\left(\mathcal{E}_{2}^{-1}(Y C)\right)=\left(\mathcal{E}_{1} \circ \mathcal{E}_{2}\right)^{-1}(Y C)
$$

and exit.

Example 9 [For $k=12, r=8$ and $p=8$ (Continued)]: Suppose

$$
\begin{aligned}
\mathcal{E}_{2}(X)=Y C & =X^{(46)} C_{4,19} \\
& =\underline{1100010 \underline{11001}} 01100101 \in \mathcal{S N}(20,2)
\end{aligned}
$$

is given as input to decoding Algorithm 6. It executes as follows.

S0: compute the index $h_{b a l} \in[0, p-1]$ such that

$$
C=01100101 \in \Gamma_{h_{b a l}} \text {. }
$$

Perform steps S0.1, S0.2 and S0.3.

S0.1: compute $\mu_{1}=m_{1}(01100101)=19$.

S0.2: compute the lexicographic index (see Table I)

$$
\xi_{19}(01100101)=4 \in[0, s(8,4,19)) .
$$

S0.3: set $h_{b a l}=\xi_{19}(C)=4 \in[0,7]$.

S1: compute the word $X=\mathcal{E}_{2}^{-1}(Y C)=\left\langle\Gamma_{h_{b a l}}\right\rangle^{-1}(Y)$. Perform steps S1.1, S1.2.

S1.1: compute

$$
d_{\hat{h}}=d_{h_{b a l}}=46 \in[0,66)
$$

from $\hat{h}=h_{\text {bal }}=4 \in[0,7]$ with Algorithm 5 . 
S1.2: from $d_{h_{b a l}}=46$, compute the word

$$
X=\mathcal{E}_{2}^{-1}(Y C)=\left\langle\Gamma_{4}\right\rangle^{-1}\left(X^{(46)}\right)=100000101111
$$

as in [20].

S2: undo the $m_{0}$-balancing encoding chosen in step SO of Algorithm 4 on the word $X$.

S3: output the word $Z=\mathcal{E}_{1}^{-1}(X)$ and exit. Again, this can be done with a size of $O\left(r^{5}+k\right)=O(k)$ memory bits and $O\left(r^{3} \log r+k \log k\right)=O(k \log k)$ bit operations because of (3).

\section{CONCLUding REMARKS}

A new efficient method to design second-order spectral-null codes is given. These new codes are obtained by applying the refined parallel decoding scheme method for the balanced code designs in [1], [12], and [19] to the random walk method for second-order spectral-null code design in [20]. This gives new non-recursive efficient code designs which make a good use of non-second-order spectral-null check words. For this reason the proposed codes are considerably less redundant than the code designs found in the literature (which are all recursive). In particular, if $k \in 2 \mathbf{I N}$ is the length of a 1-OSN code then the new 2-OSN coding scheme has length $n=k+r \in 4 \mathbf{I N}$ with an extra redundancy, $r \in 2 \mathbf{I N}$ such that $r \simeq 2 \log _{2} k+(1 / 2) \log _{2} \log _{2} k-0.174$ check bits. Table II compares the proposed non-recursive codes with the optimal codes and also with the recursive codes given in [20]. It is also shown how these codes can be implemented with $O(k \log k)$ bit operations and $O(k)$ bit memory elements. If $k \notin 2 \mathbf{I N}$ or $r \notin 2 \mathbf{I N}$ then essentially the same design can be given to encode information into the set of words $S\left(n, \mu_{0}, \mu_{1}\right)$ for some fixed $\mu_{0}, \mu_{1} \in \mathbf{I N}$. In particular, if $k, r \in 2 \mathbf{N}+1$ and $n \in 4 \mathbf{I N}$ then efficient designs can be obtained to convert the information words to words in the set $S\left(n, n / 2, n^{2} / 2\right.$ ) (or, $S(n, n / 2, n(n+2) / 2))$. Note that this non-recursive coding scheme is very general and it can be implemented in many different ways. These may depend on $k, r, p$, the choice of the particular " $m_{1}$-balancing random walk" and the choice of the partition $\mathcal{C S}$. Also, note that the decoding can be done in parallel because the check word directly identifies the $m_{1}$-balancing function used to encode and each balancing function with its inverse is very simple to compute because they are the composition of a partial reversion of a $k$-bit word and a cyclic shift. In general, parallel algorithms can be applied for both encoding and decoding as done in [19].

\section{APPENDIX}

Theorem 3: Given $r \in \mathbf{I N}$, let

$s\left(r, \mu_{0}, \mu_{1}\right) \stackrel{\text { def }}{=}\left|S\left(r, \mu_{0}, \mu_{1}\right)\right|$, for all integer $\mu_{0}, \mu_{1} \in \mathbf{I N}$.

\section{The following statements hold.}

1) Given $\mu_{0} \in[0, r]$, if $C \in S\left(r, \mu_{0}\right)$ then the minimum possible value for $m_{1}(C)$ is

$$
\alpha \stackrel{\text { def }}{=} \min _{C \in S\left(r, \mu_{0}\right)} m_{1}(C)=\frac{\mu_{0}\left(\mu_{0}+1\right)}{2} \in \mathbf{I N} .
$$

2) Given $\mu_{0} \in[0, r]$, if $C \in S\left(r, \mu_{0}\right)$ then the maximum possible value for $m_{1}(C)$ is

$$
\beta \stackrel{\text { def }}{=} \max _{C \in S\left(r, \mu_{0}\right)} m_{1}(C)=\alpha+\mu_{0}\left(r-\mu_{0}\right) \in \mathbf{I N} .
$$

3) The integer sequence

$$
\left\{s\left(r, \mu_{0}, \mu\right): r, \mu_{0}, \mu_{1} \in \mathbf{I N}\right\}
$$

satisfies the following recurrence relation.

$$
\begin{aligned}
s\left(r, \mu_{0}, \mu_{1}\right)= & s\left(r-1, \mu_{0}, \mu_{1}\right) \\
& +s\left(r-1, \mu_{0}-1, \mu_{1}-r\right),
\end{aligned}
$$

with initial conditions

$$
s\left(r, \mu_{0}, \mu_{1}\right)=\left\{\begin{array}{l}
0 \text { if } \mu_{1}<\alpha \text { or } \mu_{1}>\beta, \\
1 \text { if } \mu_{1}=\alpha \text { or } \mu_{1}=\beta ;
\end{array}\right.
$$

4) Given $\mu_{0} \in[0, r]$, the integer sequence

$$
\left\{s\left(r, \mu_{0}, \mu\right): \mu=\alpha, \alpha+1, \ldots, \beta\right\}
$$

is unimodal and symmetric with respect to

$$
\mu_{\text {mean }} \stackrel{\text { def }}{=} \frac{\alpha+\beta}{2}=\frac{\mu_{0}(r+1)}{2} \in \mathbf{R} ;
$$

namely,

$$
\begin{aligned}
& s\left(r, \mu_{0}, \alpha\right) \\
& \quad \leq s\left(r, \mu_{0}, \alpha+1\right) \leq \ldots \ldots \leq s\left(r, \mu_{0},\left\lfloor\frac{\mu_{0}(r+1)}{2}\right\rfloor\right) \\
& \quad=\max _{\mu \in[\alpha, \beta]}\left|S\left(r, \mu_{0}, \mu\right)\right| \\
& \quad=s\left(r, \mu_{0},\left\lceil\frac{\mu_{0}(r+1)}{2}\right\rceil\right) \geq \ldots \ldots \\
& \quad \geq s\left(r, \mu_{0}, \beta-1\right) \geq s\left(r, \mu_{0}, \beta\right)
\end{aligned}
$$

and

$$
s\left(r, \mu_{0}, \alpha+\mu\right)=s\left(r, \mu_{0}, \beta-\mu\right),
$$

for all integers $\mu \in\left[0, \mu_{0}\left(r-\mu_{0}\right)\right]$.

Proof: Property 1), 2) and 3) follow from [20]. Property 4) follows from [22, Th. 2].

\section{ACKNOWLEDGMENT}

The authors thank a lot the anonymous referees for their helpful comments and suggestions.

\section{REFERENCES}

[1] S. Al-Bassam and B. Bose, "On balanced codes," IEEE Trans. Inf. Theory, vol. 36, no. 2, pp. 406-408, Mar. 1990.

[2] S. Al-Bassam and B. Bose, "Design of efficient balanced codes," IEEE Trans. Comput., vol. 43, no. 3, pp. 362-365, Mar. 1994.

[3] B. Bose, "On unordered codes," IEEE Trans. Comput., vol. 40, no. 2, pp. 125-131, Feb. 1991.

[4] T. M. Cover, "Enumerative source encoding," IEEE Trans. Inf. Theory, vol. 19, no. 1, pp. 73-77, Jan. 1973.

[5] K. A. S. Immink, "Spectrum shaping with $\mathrm{DC}^{2}$-constrained channel codes," Philips J. Res., vol. 40, no. 1, pp. 40-53, 1985.

[6] K. A. S. Immink, Codes for Mass Data Storage Systems, 2nd ed. Eindhoven, The Netherlands: Shannon Foundation Publishers, 2004.

[7] D. E. Knuth, "Efficient balanced codes," IEEE Trans. Inf. Theory, vol. 32, no. 1, pp. 51-53, Jan. 1986. 
[8] R. Mascella, D. Pelusi, L. Pezza, S. Elmougy, L. G. Tallini, and B. Bose, "On efficient second-order spectral-null codes using sets of $m_{1}$-balancing functions," in Proc. IEEE Int. Symp. Inf. Theory (ISIT), Istanbul, Turkey, Jul. 2013, pp. 141-145.

[9] R. Mascella and L. G. Tallini, "On efficient high-order spectral-null codes over the $m$-ary alphabet," J. Discrete Math. Sci. Cryptogr., vol. 8, no. 3, pp. 459-481, 2005.

[10] R. Mascella and L. G. Tallini, "Efficient $m$-ary balanced codes which are invariant under symbol permutation," IEEE Trans. Comput., vol. 55, no. 8, pp. 929-946, Aug. 2006.

[11] D. Pelusi, L. G. Tallini, and B. Bose, "On $m$-ary balanced codes with parallel decoding," in Proc. IEEE Int. Symp. Inf. Theory (ISIT), Austin, TX, USA, Jun. 2010, pp. 1305-1309.

[12] D. Pelusi, S. Elmougy, L. G. Tallini, and B. Bose, " $m$-ary balanced codes with parallel decoding," IEEE Trans. Inf. Theory, vol. 61, no. 6, pp. 3251-3264, Jun. 2015.

[13] L. Pezza, L. G. Tallini, and B. Bose, "Variable length unordered codes," IEEE Trans. Inf. Theory, vol. 58, no. 2, pp. 548-569, Feb. 2012.

[14] R. M. Roth, P. H. Siegel, and A. Vardy, "High-order spectral-null codes-Constructions and bounds," IEEE Trans. Inf. Theory, vol. 40, no. 6, pp. 1826-1840, Nov. 1994.

[15] V. Skachek, T. Etzion, and R. M. Roth, "Efficient encoding algorithm for third-order spectral-null codes," IEEE Trans. Inf. Theory, vol. 44, no. 2, pp. 846-851, Mar. 1998

[16] T. G. Swart and J. H. Weber, "Efficient balancing of $q$-ary sequences with parallel decoding," in Proc. IEEE Int. Symp. Inf. Theory (ISIT), Seoul, South Korea, Jun./Jul. 2009, pp. 1564-1568.

[17] L. G. Tallini, S. Al-Bassam, and B. Bose, "Feedback codes achieving the capacity of the Z-channel," IEEE Trans. Inf. Theory, vol. 54, no. 3, pp. 1357-1362, Mar. 2008.

[18] L. G. Tallini, R. M. Capocelli, and B. Bose, "Design of some new efficient balanced codes," IEEE Trans. Inf. Theory, vol. 42, no. 3, pp. 790-802, May 1996.

[19] L. G. Tallini and B. Bose, "Balanced codes with parallel encoding and decoding," IEEE Trans. Comput., vol. 48, no. 8, pp. 794-814, Aug. 1999.

[20] L. G. Tallini and B. Bose, "On efficient high-order spectral-null codes," IEEE Trans. Inf. Theory, vol. 45, no. 7, pp. 2594-2601, Nov. 1999.

[21] L. G. Tallini and U. Vaccaro, "Efficient $m$-ary balanced codes," Discrete Appl. Math., vol. 92, no. 1, pp. 17-56, Mar. 1999.

[22] L. G. Tallini, "Geometric properties of high-order spectral-null codes," Italian J. Pure Appl. Math., no. 14, pp. 149-176, 2003.

[23] L. G. Tallini, "Bounds on the capacity of the unidirectional channels," IEEE Trans. Comput., vol. 54, no. 2, pp. 232-235, Feb. 2005.

[24] L. G. Tallini, S. Elmougy, and B. Bose, "Analysis of plain and diversity combining hybrid ARQ protocols over the $\mathrm{m}(\geq 2)$-ary asymmetric channel," IEEE Trans. Inf. Theory, vol. 52, no. 12, pp. 5550-5558, Dec. 2006.

[25] L. G. Tallini and B. Bose, "On $L_{1}$ metric asymmetric/unidirectional error control codes, constrained weight codes and $\sigma$-codes," in Proc. IEEE Int. Symp. Inf. Theory (ISIT), Istanbul, Turkey, Jul. 2013, pp. 694-698.

[26] C. N. Yang, "Efficient encoding algorithm for second-order spectralnull codes using cyclic bit shift," IEEE Trans. Comput., vol. 57, no. 7, pp. 876-888, Jul. 2008 .

[27] C. N. Yang, Z. Y. Lin, and S. L. Peng, "Reducing code length of second-order spectral-null code," IEEE Trans. Comput., vol. 64, no. 2, pp. 492-503, Feb. 2015.

[28] J.-H. Youn and B. Bose, "Efficient encoding and decoding schemes for balanced codes," IEEE Trans. Comput., vol. 52, no. 9, pp. 1229-1232, Sep. 2003

[29] J. H. Weber and K. A. S. Immink, "Knuth's balanced codes revisited," IEEE Trans. Inf. Theory, vol. 56, no. 4, pp. 1673-1679, Apr. 2010.

Luca G. Tallini was born in Frascati (Rome), Italy. He received the Laurea in Mathematics magna cum laude from the University of Rome "La Sapienza" in 1991, the M. S. and Ph. D. degrees in Computer Science from Oregon State University, Corvallis OR, in 1994 and 1996 respectively. From June 1997 to June 1998 he had been with the Dipartimento di Informatica ed Applicazioni, University of Salerno, Italy. From June 1998 to December 2002, he had been Assistant Professor at Politecnico di Milano, Italy. Since 2003 he has been with the University of Teramo, Italy, where he is a Professor at the Faculty of Communication Science and an Accademic Senator for the scientific research. His research interests include coding theory, combinatorics, combinatorial algorithms, combinatorial geometry, fault-tolerant computing, parallel computing, neural networks, and VLSI.
Danilo Pelusi received the $\mathrm{Ph}$. D. degree in Computational Astrophysics from the University of Teramo, Teramo, Italy, in 2006. He is with the Faculty of Communication Sciences, University of Teramo, Teramo, Italy. His current research interests include information theory, fuzzy logic and genetic algorithms.

Raffaele Mascella received the Laurea degree in mathematics from the University of L'Aquila in 1995 and the $\mathrm{PhD}$ degree from University of Teramo in 2004. From March 2005 to December 2012, he was an assistant professor; since December 2012, he has been an associate professor and the vice-dean of the Faculty of Communication Sciences at the University of Teramo, Italy. His research interests include coding theory, combinatorics, fuzzy logic, neural networks, philosophy of computing and philosophy of mathematics.

Laura Pezza was born in Frosinone, Italy. She received the Laurea degree in Mathematics magna cum laude from the University of Rome "La Sapienza" in 1991 and the Dottorato di Ricerca (Ph. D.) degree in Applied Mathematics from the University of Florence in 1996. From September 1996 she has been with the Dipartimento di Scienze di Base ed Applicate per l'Ingegneria, University of Rome "La Sapienza", where she is an Assistant Professor of Numerical Analysis. His research interests include applied mathematics, fluid dynamic, wavelets and refinable functions, signal analysis, and coding theory.

Samir Elmougy received the $\mathrm{Ph}$. D degree in computer science (2005) from the School of Electrical Engineering and Computer Science, Oregon State University, USA; the M. Sc. degree in computer science (1996) and the B. Sc. degree in statistics and scientific computing (1993) both from Mansoura University, Egypt. He is currently an associative professor and the chair of Computer Science Department, Faculty of Computers and Information, Mansoura University, Egypt. From 2008 to 2014, he had been with King Saudi University, Riyadh, Saudi Arabia as an assistant professor of Computer Science at the College of Computers and Information Sciences. His current research interests include algorithms, error correcting codes, computer networks, software engineering and machine learning.

Bella Bose (S'78-M'80-SM'94-F'95) received the B. E. degree in electrical engineering from Madras university, India in 1973; the M. E. degree in electrical engineering from the Indian Institute of Science, Bangalore, in 1975; and the $\mathrm{M}$. S. and Ph. D. degrees in computer science and engineering from Southern Methodist University, Dallas, Texas, in 1979 and 1980, respectively. Since 1980, he has been with Oregon State University where he is a Professor and the Senior Associate Head of the School of Electrical Engineering and Computer Science. His current research interests include error control codes, fault-tolerant computing, parallel processing, and computer networks. He is a fellow of both the ACM and the IEEE. 